\title{
X-linked primary ciliary dyskinesia due to mutations in the cytoplasmic axonemal dynein assembly factor PIH1D3
}

Chiara Olcese ${ }^{1,2, \star}$, Mitali P. Patel $^{3, \star}$, Amelia Shoemark $^{4, \star}$, Santeri Kiviluoto ${ }^{5, \star}$, Marie Legendre ${ }^{6, \star}$, Hywel J. Williams ${ }^{7}$, Cara K. Vaughan ${ }^{8}$, Jane Hayward ${ }^{3}$, Alice Goldenberg ${ }^{9}$, Richard D. Emes ${ }^{10,11}$, Mustafa M. Munye ${ }^{3}$, Laura Dyer ${ }^{3}$, Thomas Cahill ${ }^{4}$, Jeremy Bevillard ${ }^{1}$, Corinne Gehrig ${ }^{1}$, Michel Guipponi ${ }^{1} 12$, Sandra Chantot ${ }^{6}$, Philippe Duquesnoy ${ }^{6}$, Lucie Thomas ${ }^{6}$, Ludovic Jeanson ${ }^{6}$, Bruno Copin ${ }^{6}$, Aline Tamalet ${ }^{13}$, Christel Thauvin-Robinet ${ }^{14}$, Jean- François Papon ${ }^{15}$, Antoine Garin ${ }^{15}$, Isabelle Pin ${ }^{16}$, Gabriella Vera9 ${ }^{9}$, Paul Aurora ${ }^{17,18}$, Mahmoud R. Fassad ${ }^{3,19}$, Lucy Jenkins ${ }^{20}$, Christopher Boustred ${ }^{20}$, Thomas Cullup ${ }^{20}$, Mellisa Dixon ${ }^{4}$, Alexandros Onoufriadis ${ }^{21}$, Andrew Bush ${ }^{4,22,}$ Eddie M.K. Chung 23, Stylianos E. Antonarakis 1,12,24, Michael R. Loebinger ${ }^{25}$, Robert Wilson 25, Miguel Armengot ${ }^{26}$, Estelle Escudier ${ }^{6}$, Claire Hogg ${ }^{4}$, UK10K Rare Group ${ }^{\dagger}$, Serge Amselem ${ }^{6, \star \star}$, Zhaoxia Sun ${ }^{5, \star \star}$, Lucia Bartoloni ${ }^{1,27, \star \star \star}$, Jean-Louis Blouin ${ }^{1,12, \star \star} \&$ Hannah M. Mitchison ${ }^{3, \star \star}$

By moving essential body fluids and molecules, motile cilia and flagella govern respiratory mucociliary clearance, laterality determination and the transport of gametes and cerebrospinal fluid. Primary ciliary dyskinesia (PCD) is an autosomal recessive disorder frequently caused by non-assembly of dynein arm motors into cilia and flagella axonemes. Before their import into cilia and flagella, multi-subunit axonemal dynein arms are thought to be stabilized and pre-assembled in the cytoplasm through a DNAAF2DNAAF4-HSP9O complex akin to the HSP90 co-chaperone R2TP complex. Here, we demonstrate that large genomic deletions as well as point mutations involving PIH1D3 are responsible for an X-linked form of PCD causing disruption of early axonemal dynein assembly. We propose that PIH1D3, a protein that emerges as a new player of the cytoplasmic pre-assembly pathway, is part of a complementary conserved R2TP-like HSP9O co-chaperone complex, the loss of which affects assembly of a subset of inner arm dyneins.

\footnotetext{
${ }^{1}$ Department of Genetic Medicine and Development, University of Geneva School of Medicine, CH-1211 Geneva, Switzerland. ${ }^{2}$ Department of Life Sciences and Biotechnologies, University of Ferrara, 46-44121 Ferrara, Italy. ${ }^{3}$ Genetics and Genomic Medicine, University College London (UCL) Great Ormond Street Institute of Child Health, Guilford Street, London WC1N 1EH, UK. ${ }^{4}$ Paediatric Department, Royal Brompton Hospital, Sydney Street, London SW3 6NP, UK. ${ }^{5}$ Department of Genetics, Yale University School of Medicine, 333 Cedar Street, New Haven, Connecticut 06520, USA. ${ }^{6}$ Sorbonne Universités, UPMC Univ Paris 06, INSERM UMR_S933 and Service de Génétique et Embryologie Médicales, Hôpital Armand-Trousseau, AP-HP, Paris 75012, France. ${ }^{7}$ GOSgene, Genetics and Genomic Medicine Programme, University College London (UCL) Great Ormond Street Institute of Child Health, 30 Guilford Street, London WC1N 1EH, UK. ${ }^{8}$ Institute of Structural and Molecular Biology, University College London and Birkbeck College, Biological Sciences, Malet Street, London, WC1E 7HX, UK. ${ }^{9}$ Service de Génétique, CHU de Rouen, INSERM U1079, Université de Rouen, Centre Normand de Génomique Médicale et Médecine Personnalisée, Rouen, France. ${ }^{10}$ School of Veterinary Medicine and Science, University of Nottingham, Sutton Bonington Campus, Leicestershire LE12 5RD, UK. ${ }^{11}$ Advanced Data Analysis Centre, University of Nottingham, Sutton Bonington Campus, Leicestershire LE12 5RD, UK. ${ }^{12}$ Department of Genetic Medicine and Laboratory, University Hospitals of Geneva, CH-1211 Geneva, Switzerland. ${ }^{13}$ Service de Pneumologie Pédiatrique, Centre National de Référence des Maladies Respiratoires Rares, Hôpital Armand-Trousseau, AP-HP, Paris 75012, France. ${ }^{14}$ Centre de génétique, CHU Dijon Bourgogne, Équipe EA4271 GAD, Université de Bourgogne, Hôpital François Mitterrand, 21000 Dijon, France. ${ }^{15}$ Service d'Oto-Rhino-Laryngologie et de Chirurgie Cervico-Maxillo-Faciale, Hôpital Bicêtre, AP-HP, Le Kremlin-Bicêtre 94275, France. ${ }^{16}$ Pédiatrie, CHU Grenoble Alpes, INSERM U 1209, Institut for Advanced Biosciences, Université Grenoble Alpes, Grenoble, France. ${ }^{17}$ Department of Paediatric Respiratory Medicine, Great Ormond Street Hospital for Children, London WC1N 3JH, UK. ${ }^{18}$ Department of Respiratory, Critical Care and Anaesthesia Unit, University College London (UCL) Great Ormond Street Institute of Child Health, Guilford Street, London WCIN 1EH, UK. ${ }^{19}$ Human Genetics Department, Medical Research Institute, Alexandria University, El-Hadra Alexandria 21561, Egypt. ${ }^{20}$ North East Thames Regional Genetics Laboratory, Great Ormond Street Hospital for Children NHS Foundation Trust, Queen Square, London WC1N 3BH, UK. ${ }^{21}$ Department of Medical and Molecular Genetics, Division of Genetics and Molecular Medicine, King's College London School of Medicine, Guy's Hospital, London SE1 9RT, UK. ${ }^{22}$ Department of Paediatric Respiratory Medicine, National Heart and Lung Institute, Imperial College London, London SW3 6LR, UK. ${ }^{23}$ Population, Policy and Practice, University College London (UCL) Great Ormond Street Institute of Child Health, Guilford Street, London WC1N 1EH, UK. ${ }^{24}$ Institute of Genetics and Genomics in Geneva, iGE3, CH-1211 Geneva, Switzerland. ${ }^{25}$ Host Defence Unit, Respiratory Medicine, Royal Brompton Hospital, London SW3 6NP, UK. ${ }^{26}$ Rhinology and Primary Ciliary Dyskinesia Unit, General and University Hospital, Medical School, Valencia University, Valencia E-46014, Spain. ${ }^{27}$ UOSD Laboratorio Analisi Venezia, ULSS12 Veneziana, 30121 Venezia, Italy. * These authors contributed equally to this work. ${ }^{* \star}$ These authors jointly supervised this work. Correspondence and requests for materials should be addressed to H.M.M. (email: h.mitchison@ucl.ac.uk).

$\dagger$ f full list of consortium members appears at the end of the paper.
} 
n mammals, motile cilia are abundant (200-300 per cell) in multiciliated cells lining various specialized epithelia, including the airways, the oviduct and ependyma. The motile cilium is highly similar in structure to the motile flagella of sperm, being a long cellular projection sustained by a complex cytoskeleton structure (axoneme) composed of $>200$ proteins. Motile cilia axonemes are composed of nine peripheral microtubule doublets surrounding two central microtubules $(9+2)$ except for motile cilia of the embryonic node, which lack the central apparatus $(9+0)$. Several other structures attach along the length of the microtubules at 96-nm intervals to coordinate axonemal beating, including ATP-hydrolysing motor protein complexes called the outer dynein 'arms' (ODAs) and inner dynein arms (IDAs), as well as radial spokes and nexin-dynein regulatory complexes ${ }^{1}$.

Structural or ciliogenesis defects that affect cilia motility cause primary ciliary dyskinesia (PCD), a generally autosomal recessive disorder affecting 1 per 10,000-15,000 live births $s^{2,3}$. Typical features are chronic and progressive respiratory symptoms arising from deficient mucociliary clearance of the airways, manifesting in diffuse congestive lung disease and bronchiectasis. Since sperm flagella are structurally similar to cilia, PCD has a high rate of male infertility. Laterality defects arising from dysmotile nodal cilia affect half of patients, causing situs inversus or often more complex heterotaxies and congenital heart defects ${ }^{4}$. PCD is clinically and genetically heterogeneous with mutations in $>30$ genes causing disease ${ }^{2}$. A common defect is loss of both ODAs and IDAs from the ciliary axonemes, due to mutations in genes encoding axonemal dynein assembly ('DNAAF') and attachment factors including DNAAF1/LRRC50 (refs 5,6), DNAAF2/KTU 7 , DNAAF3 (ref. 8), DNAAF4/DYX1C1 (ref. 9), DNAAF5/ HEATR2 (refs 10,11), SPAG1 (ref. 12), C21ORF59 (ref. 13), LRRC6 (refs 14,15), ZMYND10 (refs 16,17) and CCDC103 (refs 18,19). The joint loss of the ODAs and IDAs is one of the most variable ultrastructural defects underlying PCD since affected individuals often have partial defects and truncated dynein arms $7,9,12,13,16,19$.

The assembly of dynein arms into cilia is a highly evolutionarily conserved mechanism within the cytoplasm. Dynein components synthesized in the cell body are pre-assembled into multi-subunit 'arm' structures, which are transported by intraflagellar transport (IFT) into the axoneme for microtubule attachment ${ }^{20,21}$. The stabilization, folding and pre-assembly of the dynein arm motors is governed by a cytoplasmic chaperone-mediated protein network ${ }^{20,22,23}$. ODA assembly is thought to involve chaperone-mediated attachment of intermediate chain (IC) dyneins DNAI1 and DNAI2 to heavy chain (HC) dyneins (DNAH5, DNAH11). This IC-HC assembly is facilitated by DNAAF1, DNAAF2 and DNAAF3, with DNAAF3 probably acting at the final stages of chaperone dissociation $^{8}$. DNAAF2 binds at least one IC (DNAI2), heat shock protein (HSP) 70 (ref. 7), as well as DNAAF4 which itself can bind both HSP70 and HSP90 (refs 9,24). DNAAF2-DNAAF4 were recently described as an R2TP-like co-chaperone complex, similarly recruited to HSP90 for assembly and regulation of multi-subunit complexes ${ }^{25,26}$. Other cytoplasmic axonemal dynein assembly factors are also emerging to have likely chaperone functions, for example C21ORF59 (ref. 27).

Here, by studying the molecular basis of PCD in unexplained cases, we have identified a new player in this cytoplasmic pre-assembly pathway, mutations in which cause X-linked disease and a variable loss of cilia dynein arms in both human PCD and the corresponding zebrafish PCD model. From structural, functional and clinical electron tomography data, we propose this might act in a complementary module akin to the DNAAF2-DNAAF4-HSP90 co-chaperone complex.

\section{Results}

PIH1D3 mutations cause PCD. A number of approaches were used by three molecular genetics laboratories (London, Geneva and Paris) to identify novel mutations causing PCD: next-generation sequencing (NGS) of whole exomes of affected individuals from 76 families, a targeted gene panel in affected individuals from 133 families and whole-genome SNP-array analyses in a male patient with intellectual disability and two male siblings from a consanguineous union. The identification of putative mutations in the $\mathrm{X}$-linked gene PIH1D3 in different patients prompted us to screen this gene by Sanger sequencing in a cohort of 32 independent male patients; this targeted screening identified a high proportion of affected families, with four males from three families carrying PIH1D3 mutations $(9.5 \%(3 / 32)$ of independent cases screened). Overall, these studies led to the identification of PIH1D3 molecular defects in affected males from nine independent families, which were all family-unique (Fig. 1a; Supplementary Figs 1 and 2): three nonsense mutations (c.127G > T, p.Glu43* in PCD12 II:1; c.266G > A, p.Trp89* in PCD392 II:1; c.511C > T, p.Gln171* in DCP1218), two frameshift mutations (c.263_268delinsG, p.Ile88Argfs ${ }^{\star} 12$ in DCP894; c.489_492del; p.Ile164Leufs ${ }^{\star} 11$ in GVA30 II:1), one missense change (c.397G > T, p.Asp133Tyr in DCP68), as well as genomic deletions containing the PIH1D3 gene $(1.93-\mathrm{Mb}, 3.27-\mathrm{Mb}$ and 3.73-Mb deletions in DCP603/DCP1747, DCP1337 and DCP855, respectively). The complement of genes contained within the latter, larger deletions are listed in Supplementary Table 1. In the NGS screening no other variants of interest were detected, and all the nine identified PIH1D3 variants were unique, having never been previously reported and being absent from all available databases of normal human variation (ExAC, 1000G, EVS). The nonsense, frameshift and deletion mutations are all predicted as protein disrupting and Fig. 1b shows a high level of conservation of Asp133 which is located within a key surface domain of PIH1D3, as described further below.

The affected families are of European origin except for PCD392 from Sri Lanka and the affected brothers DCP603 and DCP1747 from the sole consanguineous family (DC393) who are from Morocco (Supplementary Fig. 1; Supplementary Table 2). Familial segregation analysis by Sanger sequencing showed inheritance consistent with recessive X-linked disease in all cases, an inheritance pattern never before shown for non-syndromic PCD (Supplementary Fig. 1). Over-expressed mutated GFP-tagged PIH1D3 showed results consistent with the expected effect on the protein (Supplementary Fig. 3). Of the total 13 affected males, 6 had situs inversus totalis which is consistent with the typical PCD randomized left-right body plan. All have classic PCD features including neonatal respiratory distress, recurrent respiratory infections, chronic cough, sinusitis, recurrent pneumonia, otitis media and bronchiectasis (Supplementary Table 2). PCD392 II:1 had developmental delay considered unrelated to PCD and the affected individuals DCP855 and DCP1337 carrying larger chromosomal deletions of $>3 \mathrm{Mb}$ affecting multiple genes also had intellectual disability. Sperm motility tested in five affected men carrying PIH1D3 mutations showed that all had static flagella (asthenozoospermia) (Supplementary Table 2).

PIH1D3 is a putative axonemal dynein assembly factor. As a first step in investigating the likely function of PIH1D3, we looked at the domain organization of the protein. PIH1D3 is predicted with high confidence not to contain a 'PIH' domain ('protein interacting with heat shock protein 90'), but rather to contain a highly conserved 'CS' fold occupying $40 \%$ of the protein (Fig. 1a; Supplementary Fig. 4). PIH and CS are structurally 
distinct domains, although found in functionally similar proteins. The CS is a bipartite protein-protein interaction domain (Interpro \#007052) so called because it is shared by CHORD (cysteine- and histidine-rich domain)-containing proteins and the HSP90 co-chaperone SGT1 (suppressor of the G2 allele of Skp1 $)^{7,9,28}$. CS domains are also found in other HSP90 co-chaperones and protein folding proteins ${ }^{29}$. In contrast, the $\mathrm{N}$-terminal domain of PIH1D3 lacks any identifiable domain and is predicted with high confidence to be disordered, that is, without fixed structure, although it contains several strongly conserved regions that might have functional importance perhaps through binding to partner proteins (Supplementary Fig. 4).

a

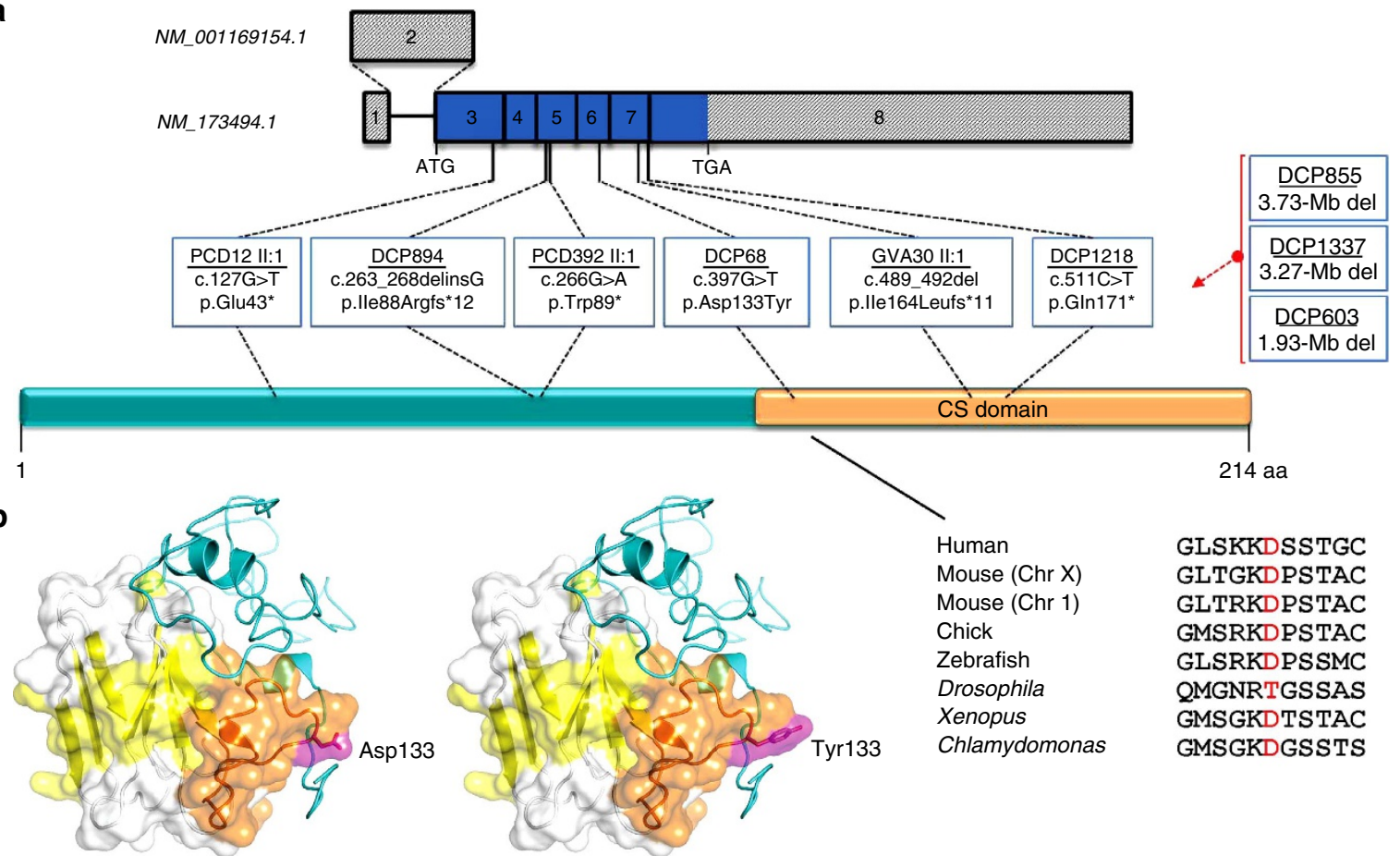

C
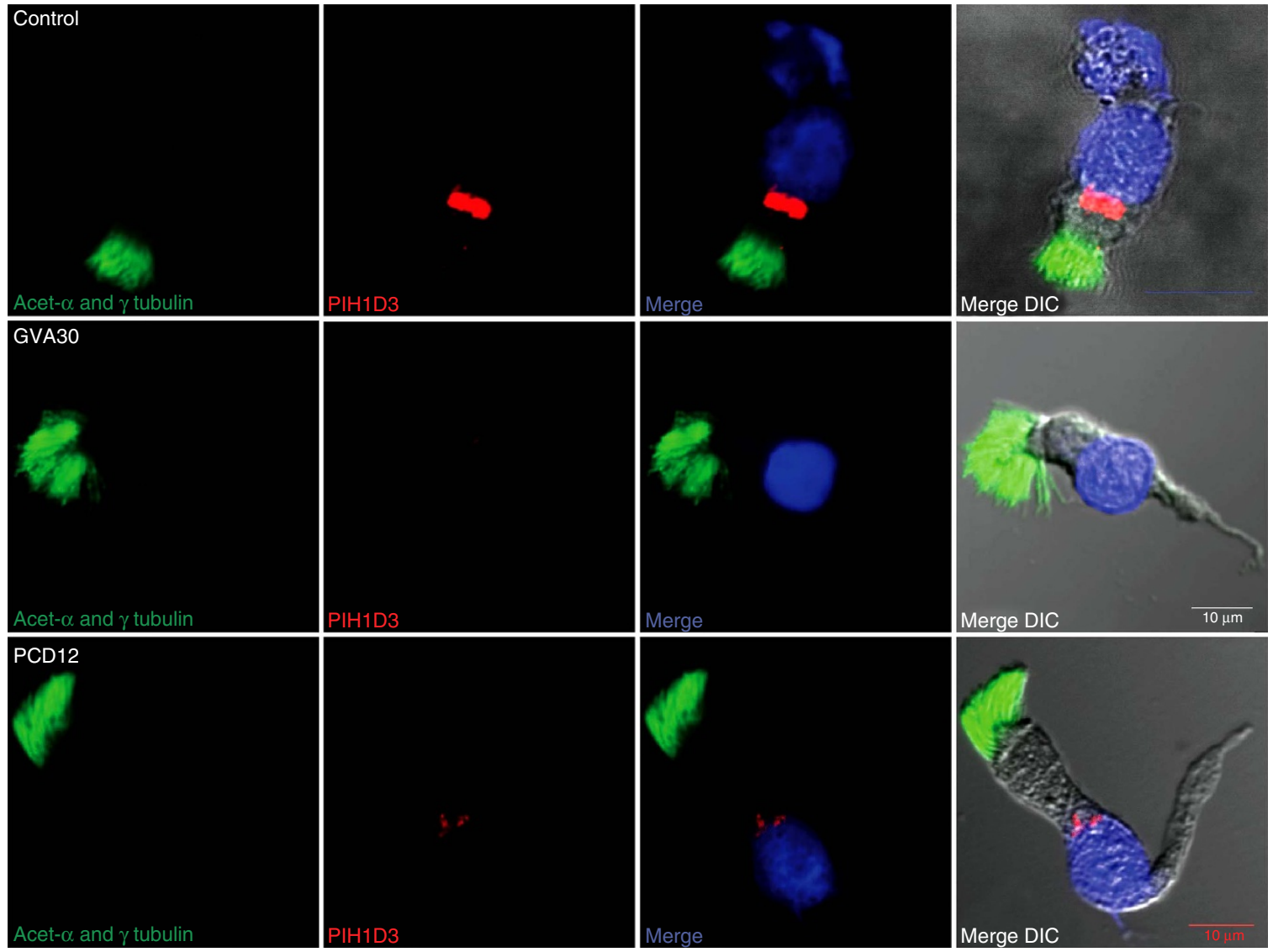
To further investigate the possible role of PIH1D3 in cilia motility, we performed cross-species BLAST analyses, showing that PIH1D3 is a highly conserved protein present only in species that have motile cilia/flagella containing dynein arms and those that require IFT for ciliary assembly (Supplementary Fig. 5). This supports a role for PIH1D3 in IFT-related dynein arm assembly. Phylogenetic evolutionary analysis showed that while humans have a single X-linked copy of PIH1D3, mice have two homologous copies located on chromosomes 1 and $\mathrm{X}$ (Supplementary Fig. 6). The intronless Chr. 1 mouse gene is most likely an expressed pseudogene recently diverged from and younger than the presumed ancient, intron-containing Chr. X gene. The proteins expressed from the murine Chr. 1 and Chr. X copy are $91 \%$ identical, differing mainly in the unstructured $\mathrm{N}$ terminus (Supplementary Fig. 4b). Knockout mice lacking the exclusively testis-expressed intronless mouse chromosome 1 pseudogene (that has been termed Pih1d3, 4930521A18Rik) have immotile sperm flagella with dynein arm defects and male sterility, but notably do not manifest situs inversus, respiratory cilia dysfunction, hydrocephalus or any ciliary phenotypes typical of $\mathrm{PCD}^{30}$. The testis-specific Pihld3 is not detectable in the mouse node at day 8 , when embryonic symmetry is first broken, and this correlates with the knockouts never showing situs inversus $^{30}$. The knockouts still express the mouse X-linked gene (called Pih1h3b, E230019M04Rik), which is expressed more widely than testis, also in the lung, brain and oviduct, but is evidently not able to rescue the sperm flagella defect arising from loss of the Chr. 1 copy $^{30}$. This evolutionary evidence shows a role for PIH1D3 in dynein arm assembly, a role that appears to have recently diverged in mice being governed by different proteins expressed in mouse sperm flagella and cilia, with the ciliaexpressed X-linked copy unable to compensate for the Chr. 1 copy in sperm. This contrasts with our data in humans showing that X-linked mutations in the sole human PIH1D3 gene cause both cilia and sperm defects.

To further investigate the likely function of PIH1D3, we determined where the protein is expressed in cells using a PIH1D3-specific antibody (demonstrated in Supplementary Fig. 3) and high-resolution immunofluorescence confocal microscopy imaging in ciliated respiratory epithelial cells from healthy controls and PIH1D3 mutation carriers. A specific localization within the cytoplasm was detected with strong staining of PIH1D3 observed in proximity to the nucleus, but no co-localization with ciliary basal body or axonemal markers ( $\gamma$-tubulin and acetylated- $\alpha$ tubulin, respectively). The cytoplasmic localization of PIH1D3 further supports its putative role in PCD as a cytoplasmic axonemal dynein assembly factor ${ }^{5-10,15}$. Notably, in cells from PIH1D3-mutated individuals GVA30 II:1 (p.Ile164Leufs ${ }^{\star} 11$ ) and PCD12 II:1 (p.Glu43*) a marked reduction of PIH1D3 protein staining was seen, with very faint staining in PCD12 II:1 and undetectable levels in GVA30 II:1 (Fig. 1c).

PIH1D3 mutations confer variable dynein arm defects. We next investigated the effect of PIH1D3 mutations at the ultrastructural level by transmission electron microscopy (TEM) of PIH1D3mutated respiratory cilia, performed within two clinical centres employing multiple replicates analysing $>300$ cilia cross-sections per replicate. This revealed in all available cases but to variable degree, a loss of both the ODAs and IDAs (Fig. 2; Supplementary Table 2). The spectrum of dynein arm loss arising from PIH1D3 mutation comprised cilia from GVA30 II:1, DCP855, DCP1337, DCP603, DCP1747, DCP894, DCP1218 and DCP68 displaying an almost complete loss of both ODA and IDA in available samples with hardly any normal cross-sections apparent, whilst PCD392 II:1 (p.Trp89*) displayed comparatively less IDA loss still with extensive loss of ODAs (Fig. 2b). However, PCD12 II:1 (p.Glu43*) appeared to be an unusual outlier, displaying much less ODA loss and primarily an IDA loss phenotype (Fig. 2b).

We proceeded to investigate the effect of PIH1D3 mutations on assembly of the ODAs and IDAs at the molecular level by immunofluorescence staining of PIH1D3-mutated respiratory cilia using antibodies directed against two established markers of human dynein arms, the dynein HC ODA marker DNAH5 and dynein light IC IDA marker DNALI1. DNAH5 staining was reduced in PIH1D3-mutated individuals, being undetectable in the axonemes of GVA30 II:1 (p.Ile164Leufs ${ }^{\star} 11$ ), corroborating with the TEM evidence of disrupted ODA assembly (Fig. 3a). In PCD12 II:1 (p.Glu43*) staining was greatly reduced as well, but some retention of DNAH5-stained ODAs was apparent, which is consistent with the TEM data shown in Fig. 2. Another ODA marker, the intermediate dynein chain DNAI2, and DNALI1 were analysed and found to display similar reduced levels in the cilia axonemes of GVA30 II:1 (p.Ile164Leufs*11) and PCD12 II:1 (p.Glu43*), again with more apparent retention of both in PCD12 II:1 (Fig. 3b). This supports the TEM findings that PCD12 II:1 retains more of the ODAs than GVA30 II:1, and also some IDAs.

The variable retention of ODAs and IDAs in PIH1D3-mutated cilia mirrors the variable effects of different mutations in some other known dynein assembly factor PCD genes ${ }^{7,9,12,13,16}$. In the case of PCD12 II:1 (p.Glu43*), in spite of the PIH1D3 nonsense mutation there appears to be less severe loss of ODAs than that of the other patients, suggesting a less severe mutation. The involvement of modifying genetic factors cannot be excluded in this individual; alternatively, the resulting PIH1D3 transcripts may escape, at least in part, degradation via the nonsensemediated mRNA decay (NMD) pathway and the protein may retain some degree of normal function. In keeping with this hypothesis, the Human Splicing Finder V3.0 tool

\section{Figure 1 | Location of X-linked PIH1D3 mutations and reduced cytoplasm-localized PIH1D3 protein in airway epithelia of affected individuals.}

(a) PIH1D3 (CXorf41) on the X chromosome has two alternate transcripts NM_001169154.1 and NM_173494.1 differing by the presence of an extra untranslated exon (exon 2) in NM_001169154.1. The open reading frame is contained in exon 3-8 (blue boxes). The PIH1D3 mutations identified are shown within the 214 amino acid protein, in relation to its sole identifiable functional domain, a CS (named after CHORD-containing proteins and SGT1) domain-spanning residue 128-214 (ScanProsite). Mutations are labelled according to the first affected male screened per family. (b) Cross-species conservation of the mutated amino acid Asp133 (D133) is shown beneath the protein, in red (right), and PHYRE-based ${ }^{63}$ three-dimensional modelling of the whole of human PIH1D3 shows a surface representation of the highly conserved C-terminal CS domain, within which the Asp133 is predicted to be a surface residue (in magenta) (left). Also highlighted are the beta sheet of the CS domain (yellow), the loops within the CS domain that join the beta strands (white) and the long loop within the CS domain on which Asp133 is located (orange). In dark blue, the disordered N terminus lacks any known structural domains. The effect of the mutated Tyr133 residue is shown (middle). (c) Immunofluorescence confocal microscopy analysis of PIH1D3 (red) in dissociated airway (nasal) epithelial cells from controls showed specific localization within the cell body in proximity to the nucleus (DAPI, blue) and no co-localization with ciliary basal body or axonemal markers ( $\gamma$-tubulin and acetylated- $\alpha$ tubulin, respectively, green) (upper panel). In cells from PIH1D3-mutated individuals GVA30 II:1 and PCD12 II:1 (middle and lower panels) there is marked reduction of PIH1D3 protein staining with undetectable levels in GVA30 II:1. Differential interference contrast (DIC) microscopy shows the outline of the cells. Scale bars, $10 \mu \mathrm{m}$. 
(http://www.umd.be/HSF3/index.html) predicts that the c.127G $>$ T mutation identified in PCD12 II:1 causes the loss of a potential exonic splice enhancer (ESE) at the $3^{\prime}$ end of exon 3 (Supplementary Fig. 7). This mutation is expected to alter splicing, especially since the MaxEntScan application ${ }^{31}$ predicts the strength of the physiological intron 3 donor splice site to be very low (that is, score of 1.07 , as compared with 7.97 to 11 for the other PIH1D3 donor splice sites). On the basis of this hypothesis, we performed RT-PCR on total RNA from a limited amount of nasal biopsy material obtained from PCD12 II:1, which confirmed the absence of exon 3 from PIH1D3 transcripts compared with those amplified from nasal biopsy of a healthy control (Supplementary Fig. 8). This indicates that the PIH1D3
c.127G $>\mathrm{T}$ mutation causes an exon skipping event in PCD12 II:1 that removes exon 3 containing the normal ATG initiation codon as well as the p.Glu43* nonsense mutation. This could allow the use of an in-frame alternative ATG initiation codon, downstream of the usual start codon located at the beginning of exon 3. The corresponding transcripts would therefore escape NMD and a shorter in-frame PIH1D3 protein could be expressed. Such an alternative ATG indeed exists in exon 4 (codon 61) and it contains a good Kozak consensus sequence as predicted by $\mathrm{ATGpr}^{32}$ (score of 0.15 versus 0.16 for the usual ATG initiation codon).

We investigated PIH1D3-mutated cilia motility using high-speed video microscopy imaging. All affected individuals

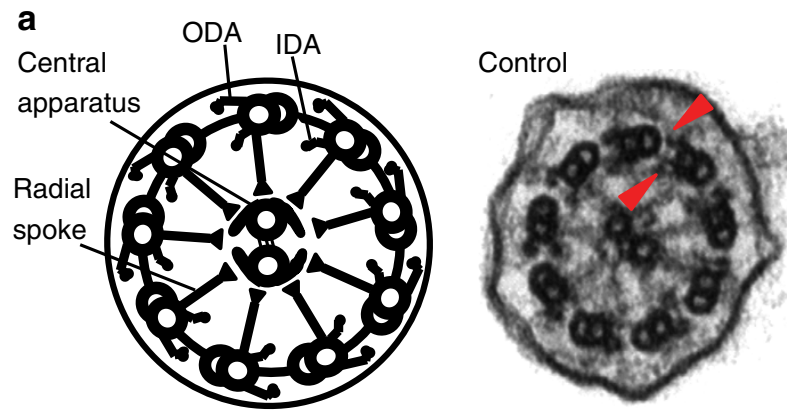

b

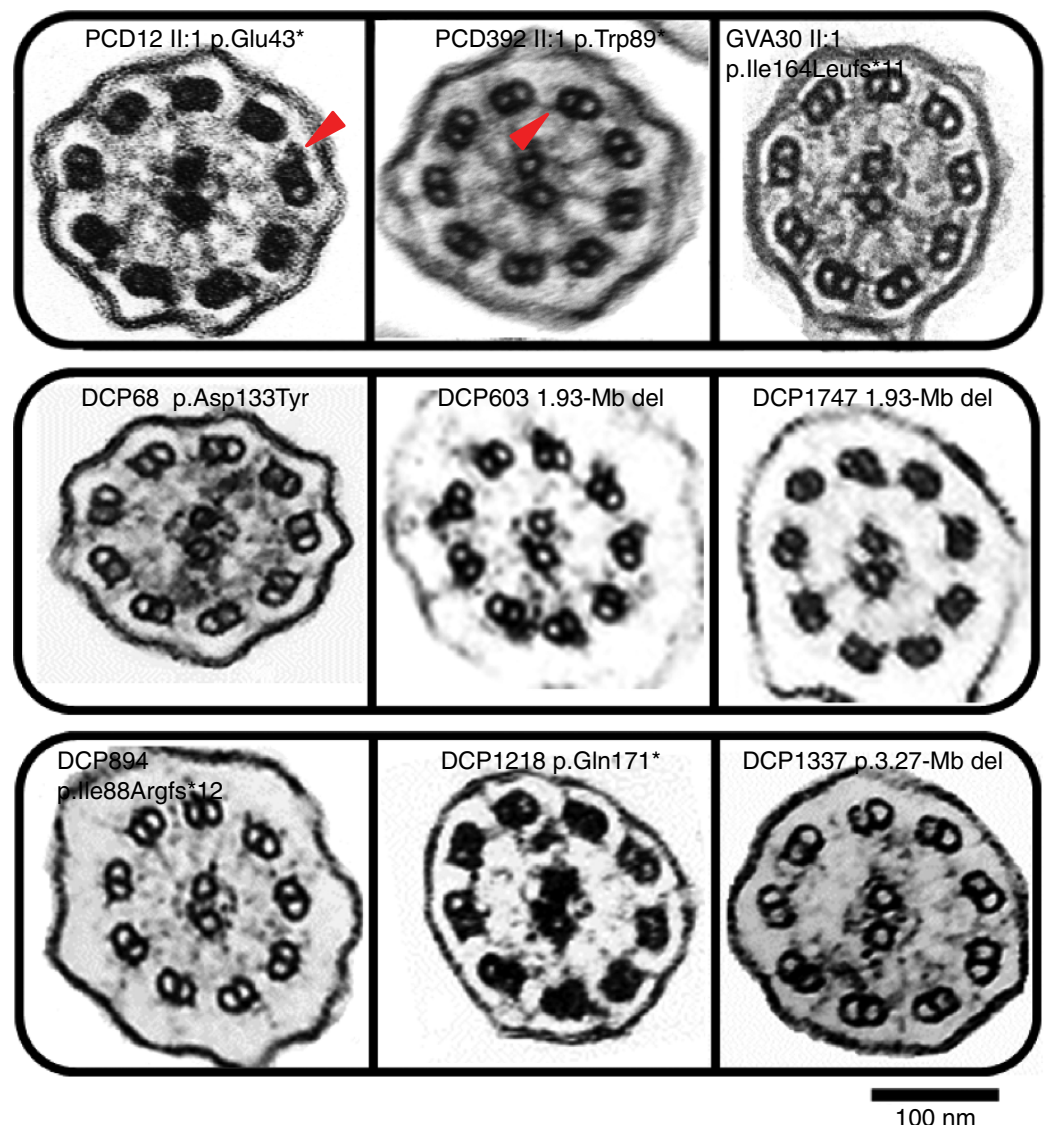

Figure 2 | PIH1D3 mutations result in non-assembly and loss of IDAs and ODAs in respiratory cilia axonemes. (a) TEM showing the cross-section arrangement of cilia from a healthy control (right) and an image (left) of the main structures of the $9+2$ motile axoneme including the outer (ODA) and inner (IDA) dynein arms (red arrowheads). (b) TEM of PIH1D3-mutant cilia from nasal respiratory epithelial cells of affected individuals display a reduction and loss of both the ODAs and IDAs. All cases show a combined loss of ODAs and IDAs but this defect can be variable and in particular mutations p.Glu43* (p.E43*, in PCD12 II:1) and p.Trp89* (p.W89*, in PCD392 II:1) are associated with some visible retention of the arms, in particular the outer (PCD12) or inner (PCD392) dynein arms (red arrowheads). In comparison, all other mutations result in the majority of cross-sections showing complete loss of both ODAs and IDAs. These images represent TEM data surveys from a minimum of 300 cross-sections per patient sample. 


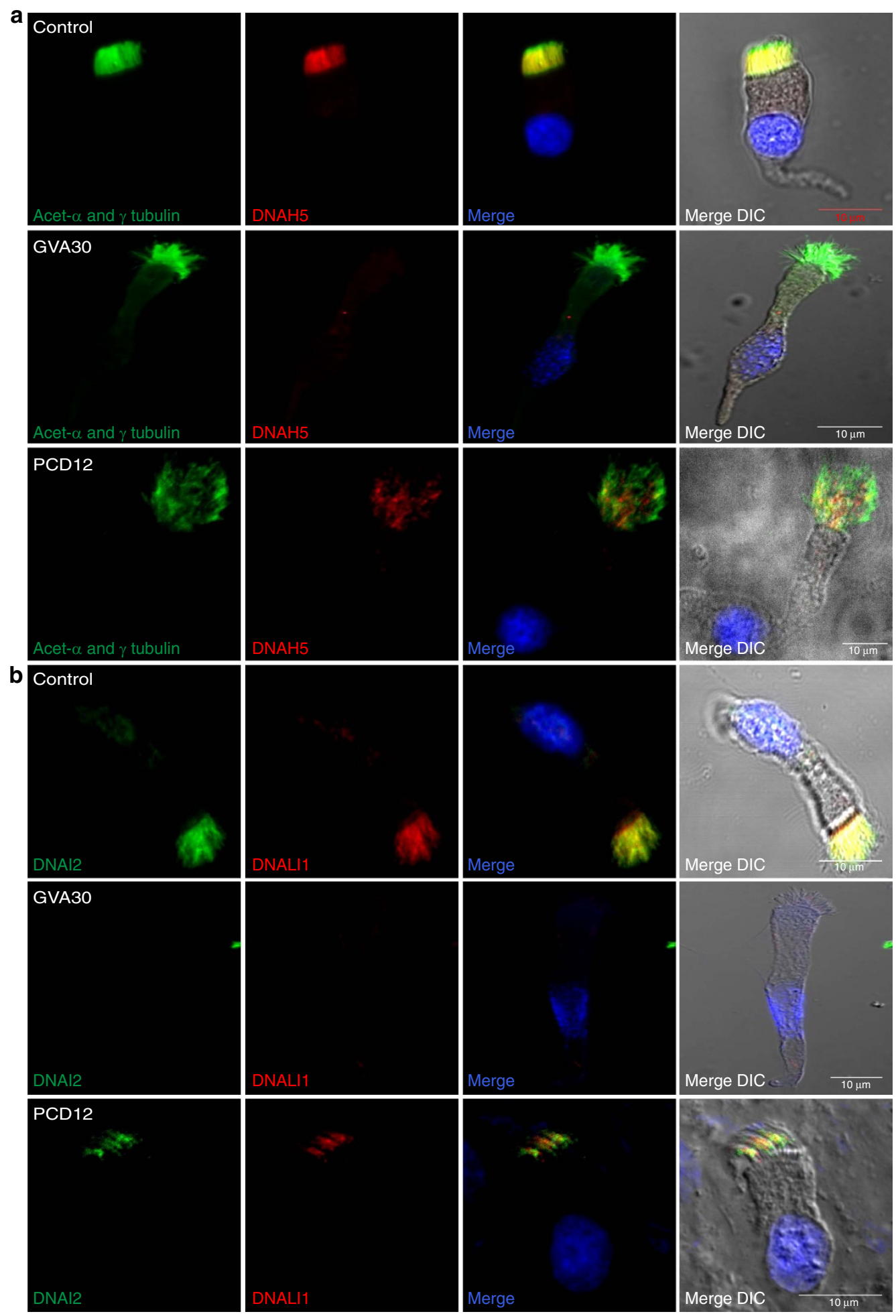

Figure 3 | PIH1D3 mutations cause defective dynein arm assembly in respiratory cilia. (a) Respiratory epithelial cells from control and PCD-affected individuals GVA30 II:1 and PCD12 II:1 carrying PIH1D3 mutations were double-labelled with antibodies directed against acetylated $\alpha$-tubulin and $\gamma$-tubulin (green) to mark the ciliary axonemes and basal bodies, and DNAH5 as a marker of the ODA (red). Both $\alpha$-tubulin and DNAH5 colocalize (yellow) along the full length of the cilia axonemes in cells from the unaffected control (upper panel). In contrast, in cells from affected individuals GVA30 II:1

(p.Ile164Leufs ${ }^{\star} 11$ ) and PCD12 II:1 (p.Glu43*) (lower panels), DNAH5 is highly reduced in the ciliary axonemes compared with $\alpha$-tubulin, being undetectable in GVA30 II:1 and markedly reduced in PCD12 II:1. (b) Respiratory epithelial cells from control and PCD-affected individuals GVA30 II:1 and PCD12 II:1 carrying PIH1D3 mutations were also double-labelled with antibodies directed against another ODA marker DNAI2 (green) and the IDA marker DNALI1 (red). Both proteins colocalize (yellow) along the full length of the cilia axonemes in cells from the unaffected control (upper panel). In contrast, in the PIH1D3 mutants, both DNAI2 and DNALI1 are reduced in the ciliary axonemes, being undetectable in GVA30 II:1 and markedly reduced in PCD12 II:1. DIC shows the cell outline. Scale bars, $10 \mu \mathrm{m}$. 
had immotile cilia consistent with dynein arm loss: as a representative example of these patients, only entirely static cilia could be seen in PCD392 II:1 (Supplementary Video 1), while in controls a healthy coordinated beating pattern was observed (Supplementary Videos 2 and 3). However in PCD12 II:1 $\left(\right.$ p.Glu43 $3^{*}$ the cilia retained some movement, displaying within the same sample a mixed beating pattern consisting of patches of static cilia alongside patches of cilia beating with normal frequency but a jittery dysmotile and disorganized pattern (Supplementary Videos 4 and 5). Since ODAs govern cilia beat frequency and propagate asymmetric waveforms, whilst IDAs regulate cilia waveform and propagation ${ }^{33,34}$, we speculate that the static cilia patches in PCD12 II:1 (p.Glu43*) correspond to those more lacking in ODAs, whilst the cilia with retained but disorganized beating could be those more lacking in IDAs.

PIH1D3 has a conserved ciliary role in other vertebrates. We further investigated PIH1D3 function using zebrafish pih1d3 ${ }^{\text {hi1392Tg/hi1392Tg }}$ mutants generated by transgenic insertion. Previous work identified a link in these mutants between loss of PIH1D3 and cystogenesis within the glomerular-tubular region of pronephric kidneys, but did not directly test for ciliary defects ${ }^{35}$. We found that zebrafish possess a single orthologous gene pih1d3 $46 \%$ identical to human PIH1D3 (Supplementary Fig. 4b). RT-PCR confirmed that pih1d3 mRNA is maternally deposited in zebrafish, and is further expressed during the first 2 days when several ciliated organs become present (Supplementary Fig. 9). hi1392Tg mutants consistently developed cysts in pronephric glomeruli and tubuli (Fig. 4a,g). This phenotype was previously linked to a lack of pronephric fluid flow caused by defective pronephric cilia motility ${ }^{36}$, and is exhibited in hi1392Tg mutants with visible pronephric dilation and dispersal of the normally closely packed pronephric cilia (Supplementary Fig. 10)

We found that cilia are virtually immotile in hi1392 Tg mutants, both in pronephric tubuli and olfactory bulbs (Fig. 4b-d; Supplementary Videos 6-9). TEM also revealed absent ODAs and IDAs in mutant cilia, thus phenocopying human PIH1D3mutated patients (Fig. 4e,f). The hil392Tg mutants additionally displayed consistent ventral tail curvature that is typical for PCD zebrafish models, and two other phenotypes considered to result from cilia dysmotility: abnormal cardiac looping presumed to arise from defective Kupffer's vesicle cilia motility ${ }^{37}$ and abnormal otolith formation caused by otic vesicle cilia dysmotility $^{38}$ (Fig. 4g). RT-PCR showed a complete lack of expression of the hi1392Tg allele (Fig. 4h), and injecting zebrafish pih1d3 mRNA completely or largely rescues the phenotypes of ventral body axis curvature, pronephric cysts and abnormal cardiac looping (Supplementary Fig. 11).

To test whether PIH1D3 is functionally conserved from zebrafish to human, we injected human PIH1D3 mRNA into embryos from crosses of heterozygous carriers of the $h i 1392 \mathrm{Tg}$ allele. Overexpression of the human protein in zebrafish appears to be detrimental. By titrating the dosage down to $10 \mathrm{pg}$, the ventral curvature phenotype is partially rescued in some of the mutant embryos (Fig. 4i-k).

PIH1D3 acts early in dynein assembly with DNAI2 interaction. We sought to further explore PIH1D3 ciliary function using the human antibody that detects a specific protein product around the Golgi region of the cytoplasm, near the nucleus (Fig. 1c). High-resolution immunofluorescence analysis revealed that PIH1D3 localizes to the trans-Golgi network in ciliated epithelia cells, with a high degree of specific co-localization between PIH1D3 and the late Golgi marker TGN-46 but much less colocalisation with the cis-Golgi marker GM-130 (Fig. 5a).
Co-localization with TGN-46 was also confirmed after overexpression of PIH1D3 in HEK293 cells (Supplementary Fig. 12). PCD12 II:1 (p.Glu43*)-ciliated airway epithelial cells did not show TGN disruption or loss of TGN-46 co-localization because of their depleted PIH1D3 protein (Fig. 5a). Thus, any retained protein expressed from this allele localizes normally. Expression of PIH1D3 compared with two other PCD genes (DNAH5, DNAI2) during ciliogenesis in cultured-ciliated human airway epithelial cells showed a similar profile, increasing before emergence of the motile cilia around day 10, with peak expression around day 24 falling to a lower plateau from day 24 . Coexpression of PIH1D3 with other PCD genes supports our proposal that it has a shared function (Fig. 5b).

To further investigate the role of PIH1D3 in dynein assembly, we examined its interactions with DNAI2, an ODA intermediate dynein chain co-assembled with another IC [DNAI1] during the first steps of cytoplasmic ODA pre-assembly ${ }^{7,30,39}$. Co-expression of GFP-tagged human PIH1D3 and Myc-tagged human DNAI2 in HEK293 cells followed by co-immunoprecipitation (IP) showed an interaction between the two proteins (Fig. 5c), which was also confirmed with swapped tags (Fig. 5d), supporting a role for PIH1D3 in axonemal dynein assembly. Since modelling predicts that the Asp133Tyr missense mutation identified in patient DCP68, which replaces a small charged residue (Asp) with a polar aromatic residue (Tyr), is within the CS in a high confidence conserved predicted surface of PIH1D3 that mediates protein-protein interactions (Fig. 1b; Supplementary Fig. 4a,b), we tested whether introduction of this missense mutation could alter the ability of PIH1D3 to interact with DNAI2. We found that the PIH1D3-DNAI2 interaction was destroyed by introduction of the missense mutation (Fig. 5c). Correspondingly, p.Asp133Tyr is predicted as 1.0 'probably damaging' using Polyphen-2 (ref. 40) and 0.05 'deleterious' according to SIFT ${ }^{41}$, consistent with a high level conservation in multiple ciliate species across Asp133 (Fig. 1b; Supplementary Fig. 4b). Similar results were obtained with three other deleterious (nonsense, frameshift) human PIH1D3 mutations identified in this study (Fig. 5c), indicating the functionally destructive nature of all four DNA variants that were modelled.

Mutations in PIH1D3 can result in partial IDA assembly. Having placed human PIH1D3 into the earliest steps of the DNAI2-ODA cytoplasmic assembly process alongside DNAAF2, a finding also supported by previous work in mice ${ }^{30}$, we next sought higher resolution understanding about the dynein arm defects in PIH1D3-mutated human cilia. We used electron tomography averaging to create a three-dimensional map of the axonemal $96 \mathrm{~nm}$ repeat containing the entire IDA, from available tissue of PCD12 II:1 (p.Glu43*) and GVA30 II:1 (p.Ile164Leufs $\left.{ }^{\star} 11\right)$. Fig. 6a,b shows a representative image of PCD12 II:1 (p.Glu43*), compared with a healthy control (Supplementary Fig. 13). There were generally few IDAs available for imaging, but we were able to detect them in some sections. In imaging of GVA30 II:1, tomograms only captured full absence of the whole IDA along with absent N-DRCs (nexin-dynein regulatory complex) and ODAs (Fig. 6d). However, imaging of PCD12 II:1 revealed three different patterns of IDA assembly in the tomograms, with ODAs being present in all imaging. The entire IDA plus N-DRC were either entirely present (Fig. 6d, PCD12 \#5-\#7), entirely absent (Fig. 6d, PCD12 \#1 and \#2) or else a partial IDA assembly was seen. Partial IDA assembly found in 2 out of 7 tomograms (Fig. 6d, PCD12 \#3 and \#4) showed a consistent pattern of loss of the $\alpha$ and $\beta$ subunits of IDA dynein I1 (also known as f) and IDA dynein $g$. Dynein d loss was undetermined, being either present or absent 
(Fig. 6a,d). Electron density mapping further confirmed the presence of all IDA and ODA components except I1/f $\alpha$, I1/f $\beta$ and the $\mathrm{d}$ and $\mathrm{g}$ subunits in tomogram \#3 (Fig. 6c). This partial
IDA assembly is consistent with Chlamydomonas data suggesting different cytoplasmic axonemal dynein assembly factors might specifically assembly distinct sets of IDA dynein subunits ${ }^{22}$.
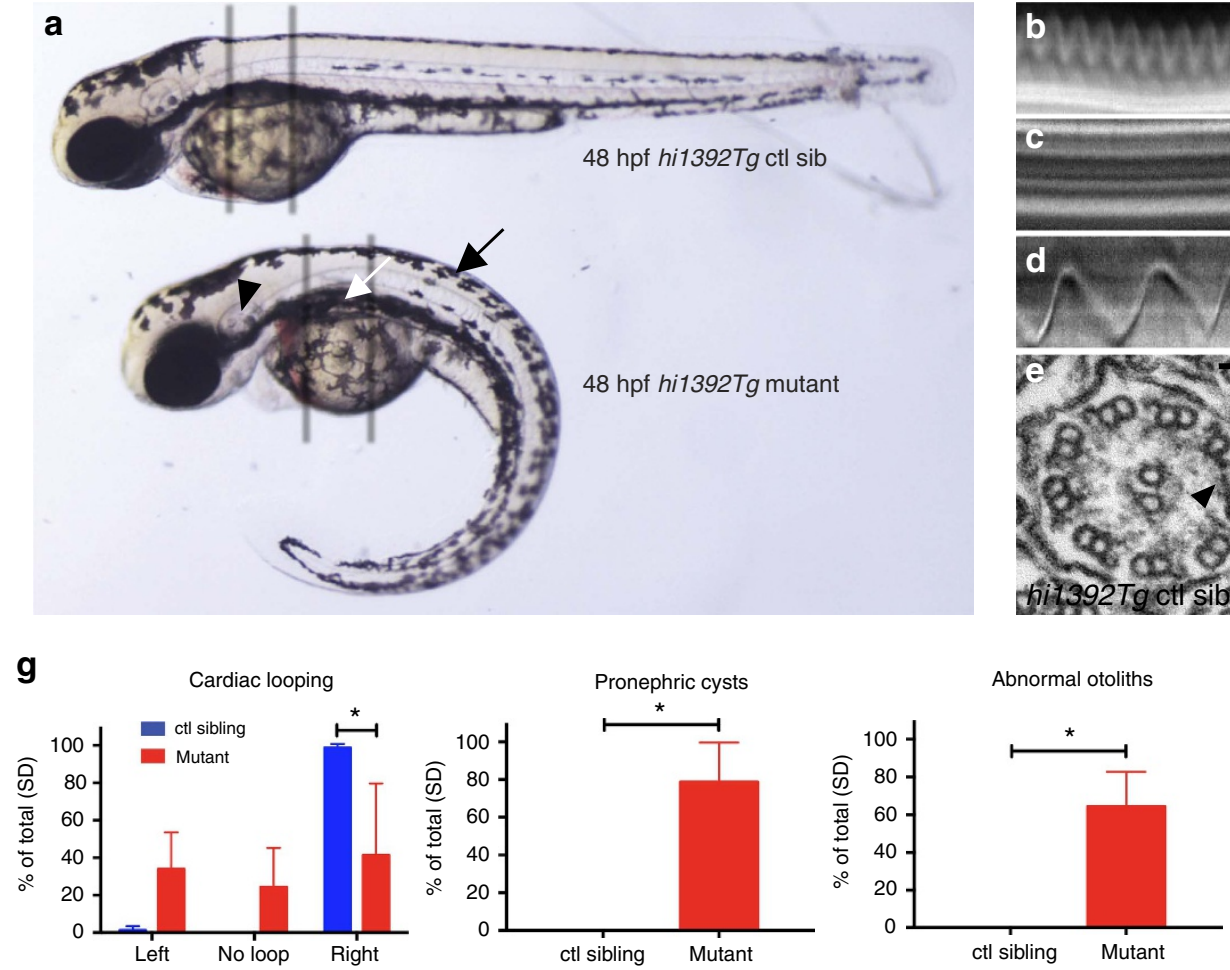

i

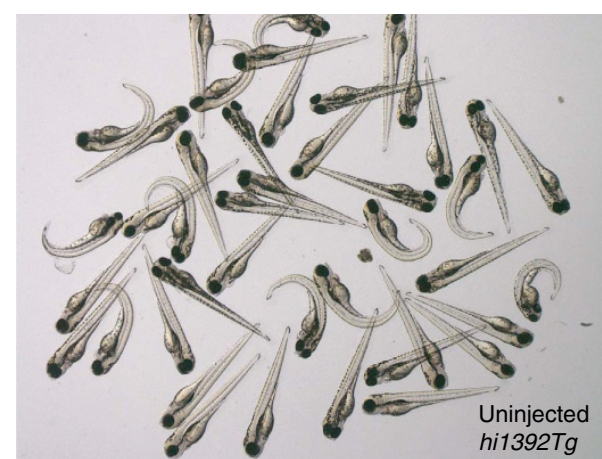

j

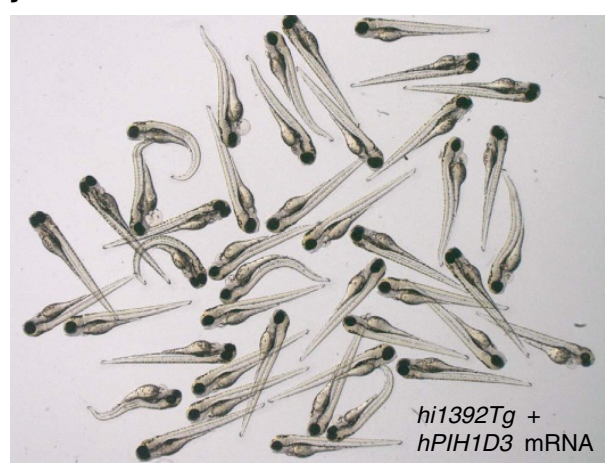

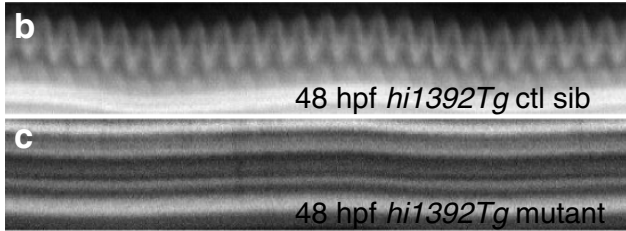
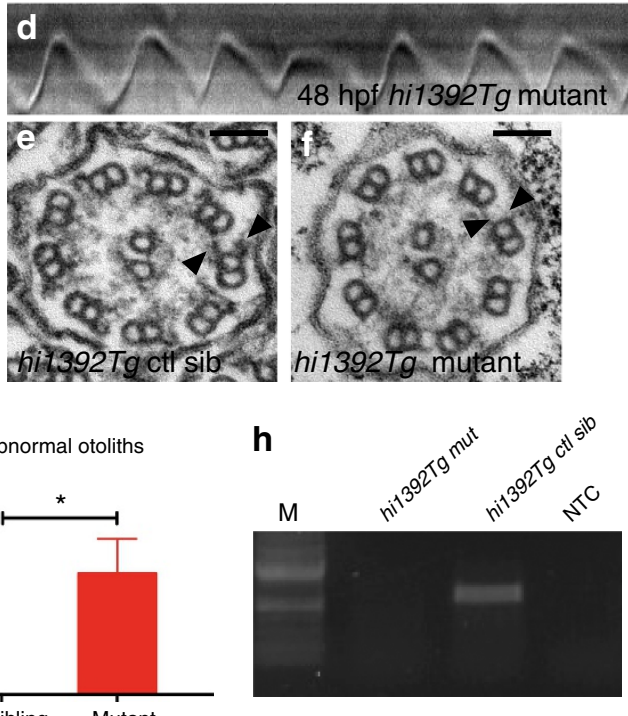

$\mathbf{k}$

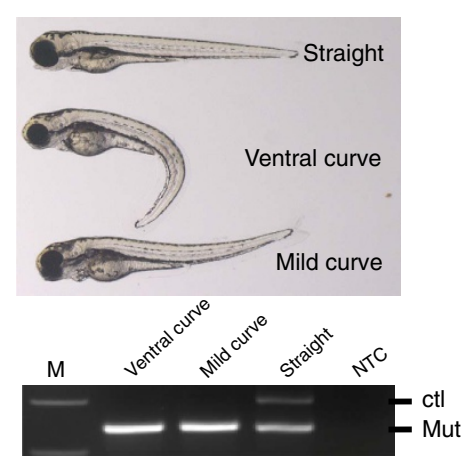

Figure 4 | Conserved function for PIH1D3 in vertebrate cilia motility and dynein arm assembly replicating the human disease. (a) Comparison of a representative hi1392 Tg control sibling (ctl sib, top), and a hi1392Tg mutant (bottom) harbouring a transgenic insertion in the pih1d3 (previously known as twister) gene, at $48 \mathrm{~h}$ post fertilization ( $48 \mathrm{hpf}$ ). Mutants display an abnormally curved body axis (black arrow), pronephric cysts (white arrow), abnormal otolith formation (black arrowhead). (b-f) Cilia defects are evident in hi1392Tg mutants at $48 \mathrm{hpf}$, with the grey bars in a highlighting areas used for pronephric cilia imaging shown in b-f. Cilia motility defects are evident in representative kymographs recorded in hi1392 Tg mutant siblings (c,d) compared with hi1392 Tg control siblings (b), created from 0.5-s, 1,000 fps videos of single pronephric cilia bundles beating in 48 hpf (representative of 16 recordings). Mutants had mainly static cilia (c) with an occasional cilia showing retained reduced beating (d). Also see Supplementary Videos 6-9. (e,f) TEM of 48 hpf pronephric cilia cross-sections from the same area reveal a reduction and loss of outer and IDAs (arrowheads) in hi1392Tg mutants (f) compared with control siblings where the arms are visible (e). Scale bar, $50 \mathrm{~nm}$. (g) Quantification of common phenotypes of hiT392Tg mutant embryos (red columns) compared with control sibling embryos (blue columns). hi7392Tg mutant embryos display $64-84 \%$ levels of abnormal (that is, absent or reverse) cardiac looping, pronephric cysts and otolith malformations, while this was never, or rarely, seen in control siblings. Otolith malformation was recorded if there were more or less than two observed. Columns represent data from three separate experiments assessing phenotype in 48 hpf embryos ( $n=40-71$ per experiment). Error bars show standard deviation with $t$-test, $P$-value ${ }^{*}<0.05$. (h) The insertion hi1392Tg creates a null allele: RT-PCR of a pool of 15 embryos indicates a complete lack of pih1d3 transcript expression in hi1392Tg mutants compared with a pool of 15 control siblings. (i-k) Examples of clutches of $72 \mathrm{hpf}$ embryos from hi1392Tg/ + crosses injected at one-cell stage with $10 \mathrm{pg}$ of human PIH1D3 mRNA show a reduction in mutant phenotypes (j) compared with non-injected hi1392Tg control siblings (i), with representative embryos with a straight, ventral curve, or mild curve phenotype shown in $\mathbf{k}$, upper panel. PCR genotyping in all three of these groups shows the lower hi1392Tg insertion allele band present, but the upper control allele band is present only in the straight-tailed siblings (k, lower panel). M, DNA size marker; NTC, non-template control. 
a
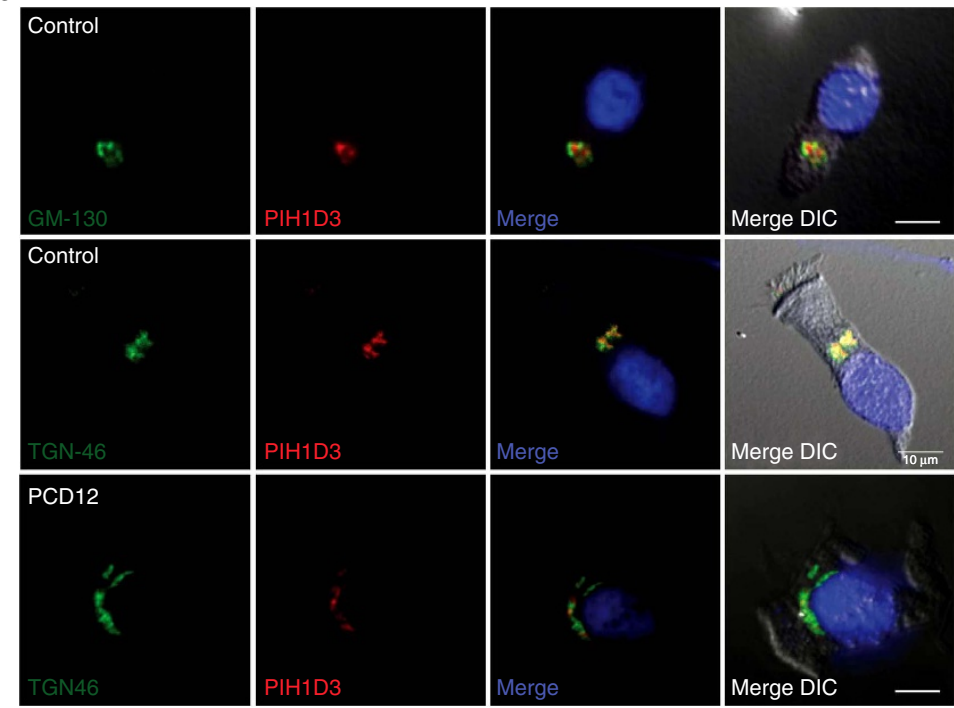

b

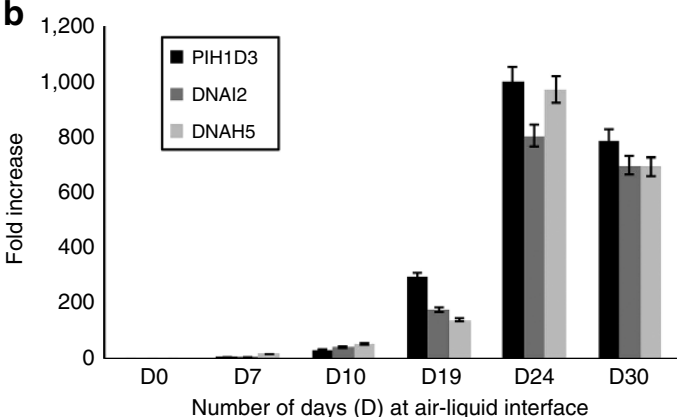

d

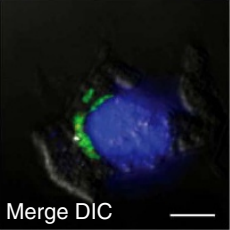

d

C

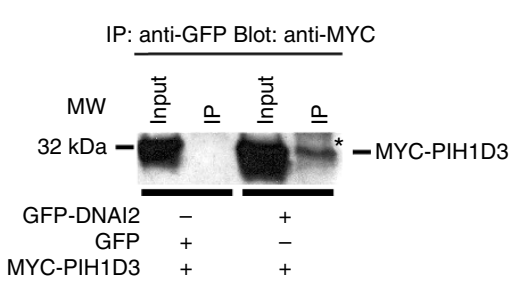
IP: anti-GFP Blot: anti-MYC

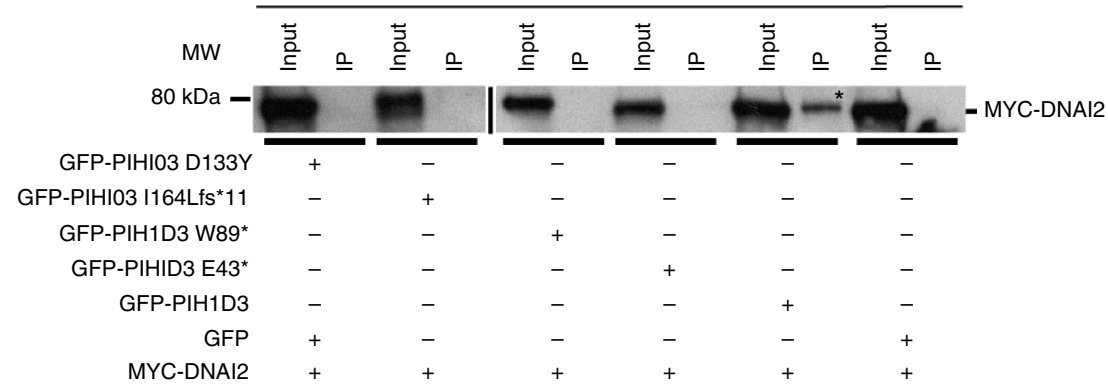

Figure 5 | Golgi-localised PIH1D3 is upregulated during ciliogenesis and forms a complex with DNAI2 destroyed by disease-causing mutations. (a) Immunofluorescence confocal microscopy analysis in dissociated airway (nasal) epithelial cells from controls shows no co-localization of PIH1D3 (red) with the cis-Golgi marker GM-130 (top panel, green). However, co-localization is seen using the trans-Golgi marker protein TGN-46 (middle panel, green), implying PIH1D3 is present within the late rather than early/medial Golgi. The affected individual PCD12 II:1 showed no gross disruption of trans-Golgi staining as a result of PIH1D3 mutation using co-localization studies of TGN-46 (green) and PIH1D3 (red) (bottom panel). Nuclei in blue (DAPI). DIC shows cell outline. Scale bars, $10 \mu \mathrm{m}$. (b) Quantitative RT-PCR analysis of PIH1D3 in normal human bronchial epithelial cells grown on an ALI to follow their progression from nonciliated basal cells to multiciliated epithelia over 30 days (D) in culture. Day (D) 0 is the first day the cells are exposed to the ALI, and typically cilia first become visible in these cultures from day 17. The qPCR analysis compared expression of PIH1D3 to the DNAH5 and DNAI2 genes, mutations in which are known to cause PCD. A similar increase in mRNA expression of all three genes was seen over time in ALI culture, from around day 10 with a peak at day 24. Around day 19, the apparent higher increase in PIH1D3 levels was not significant. The fold increase in each gene's expression was measured in triplicate, normalized at each time point to its expression at day 0. Error bars indicate s.e.m. (c) Co-IP assays were performed on human embryonic kidney (HEK293) cells co-transfected with an expression vector encoding Myc-tagged DNAI2 and either an empty GFP vector or GFP-tagged PIH1D3, corresponding either to normal full-length PIH1D3 protein or mutant PIH1D3 protein carrying mutations identified in affected individuals. Anti-GFP antibody IP was performed using a standard protocol followed by western blotting using anti-Myc antibody, showing that Myc-DNAI2 co-precipitates only with wild-type GFP-PIH1D3 and not with GFP-PIH1D3 harbouring any of the four identified disease mutations (p.Asp133Tyr/p.D133Y, p.Ile164Leufs ${ }^{\star 11 / ~}$ p.1164Lfs ${ }^{\star} 11, p . T r p 89^{*} /$ p.W89* and p.Glu43*/p.E43*). (d) A reciprocal co-transfection of Myc-tagged wild-type PIH1D3 into HEK293 cells with either empty GFP vector or GFP-tagged DNAI2 followed by anti-GFP IP and anti-Myc western blotting confirmed the co-IP between Myc-PIH1D3 and GFP-DNAI2. $\mathrm{MW}$, molecular weight marker. kDa, kilodaltons. Asterisks in $\mathbf{c}, \mathbf{d}$ indicate the immunoprecipitated proteins (labelled on the right). Uncropped blots can be found in Supplementary Fig. 14. 
a

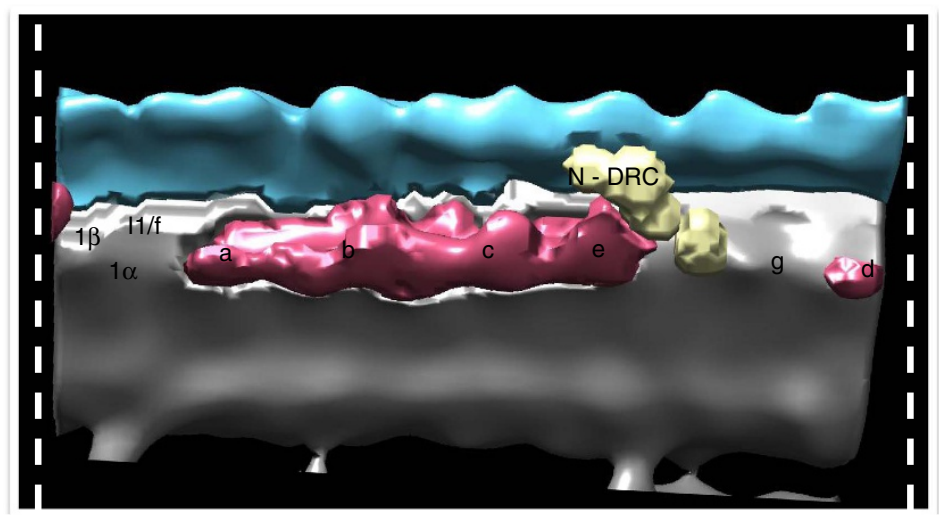

b

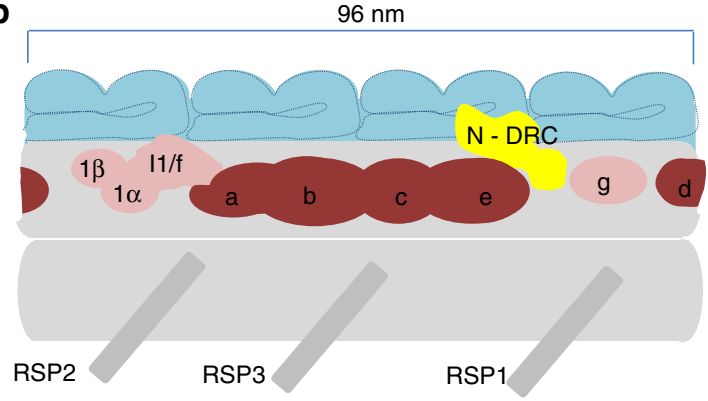

C
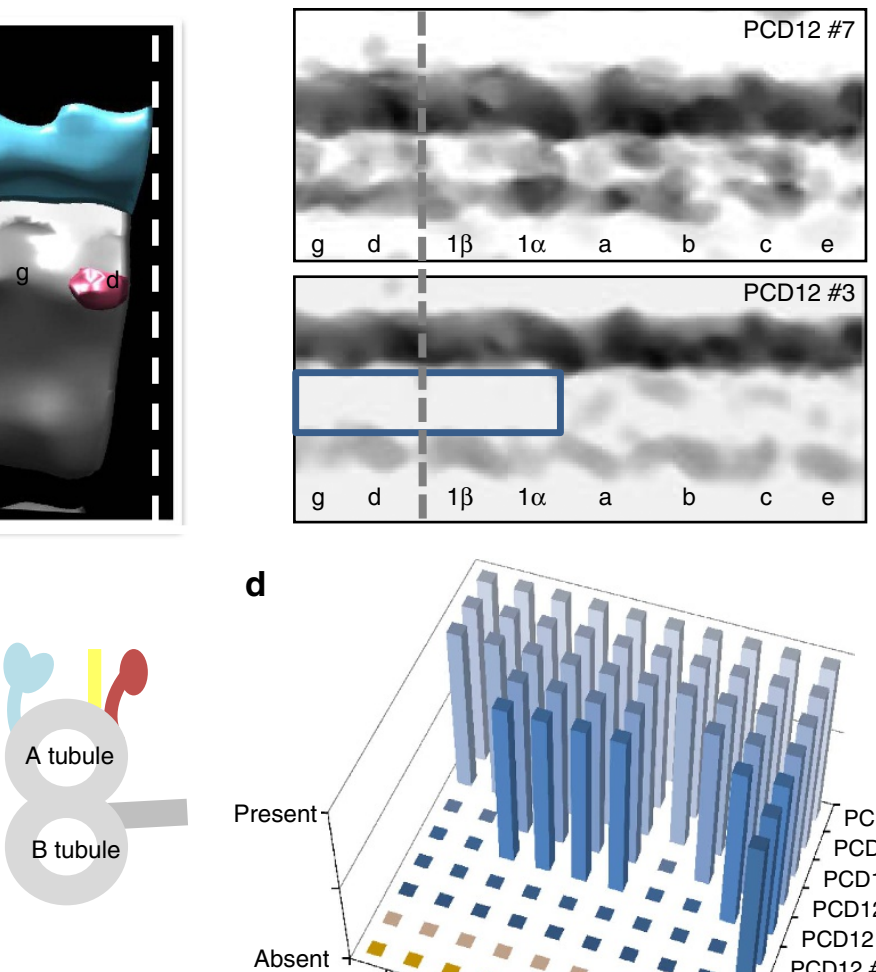

d

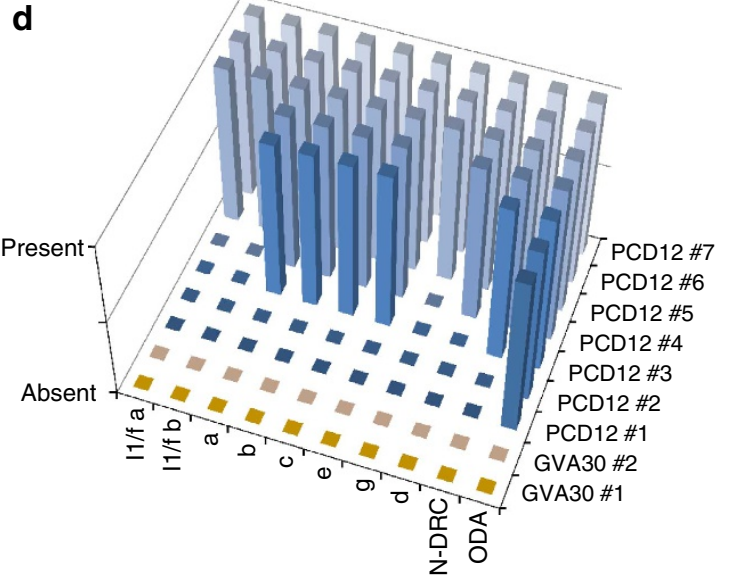

Figure 6 | PIH1D3 mutations cause a failure of specific dynein components to assemble into the IDA. (a) Electron tomography based three-dimensional map of the axonemal $96 \mathrm{~nm}$ repeat (indicated by dashed white lines) generated from an averaged tomogram (tomogram PCD12 \#4 which is also shown in d), from individual PCD12 II:1 harbouring the PIH1D3 mutation p.Glu43*. The data reconstruction displays the IDA coloured using Chimera software, showing the absence of the $\alpha$ and $\beta$ subunits of dynein 11 (also known as $\mathrm{f}$ ), in addition to dynein $\mathrm{g}$, as explained in $\mathbf{b}$. (b) A diagram of the $96 \mathrm{~nm}$ repeat of the human axoneme shown in both longitudinal (left) and transverse (right) section, based on imaging of normal tomograms (see Supplementary Fig. 13) and current published literature. ODAs are depicted in blue, IDAs in red with the inner arm dynein components absent from individual PCD12 II:1 shown in a lighter pink shade. The nexin link/dynein regulatory complex (N-DRC) is shown in yellow, with radial spoke (RSP) complexes 1, 2 and 3 and the anchoring microtubule doublets in grey. (c) Electron density analysis along the $96 \mathrm{~nm}$ repeat (boxed), showing in longitudinal section the presence of all inner and ODA components from PCD12 tomogram \#7 and the absence of the $11 / f \alpha, 11 / f \beta, d$ and g subunits (boxed) from PCD12 tomogram \#3, taken from the tomograms represented in $\mathbf{d}$. Dashed line shown for orientation compared with $\mathbf{a}$. (d) Representation of the presence or absence of IDA axonemal components in different tomograms taken from patients PCD12 II:1 (tomograms \#1-\#7) and GVA30 II:1 (tomograms \#1, \#2). IDAs were not present in the axonemes of cilia from GVA30 II:1, whilst there was more variability in PCD12 II:1 and IDA partial loss always involved non-assembly of dyneins $I 1 / f, I 1 / f$ and $g$.

\section{Discussion}

Here we report different approaches taken across three centres to identify the cause of disease in a large cohort of PCD patients. This identified protein-altering mutations in PIH1D3, a previously uncharacterized X-linked gene in humans. X-linked transmission of PCD was previously suggested ${ }^{42}$ and RPGR mutations can cause syndromic X-linked PCD with retinitis pigmentosa $^{43}$, but PIH1D3 mutations are the first reported molecular cause of X-linked non-syndromic PCD. PIH1D3 mutations may be a prominent cause of X-linked PCD, since a small targeted screen in this study for mutations in affected males with PCD due to lack of dynein arms so far unexplained at the molecular level detected PIH1D3 mutations in 9.5\% of cases.

The affected males carrying PIH1D3 mutations displayed deficient mucociliary clearance, situs inversus and asthenozoospermia, indicating that in humans the single X-linked PIH1D3 participates in axonemal dynein assembly in both the respiratory system and testis, as well as in laterality determination. In contrast mice have two PIH1D3 homologues, a testis-restricted mouse Chr. 1 Pihld 3 being essential for dynein assembly in the sperm flagellum, while mouse Chr. X Pih 1 h $3 b$ is expressed in testis and also in ciliated tissues (lung, brain, oviduct) but cannot compensate to rescue sperm motility. In the Human Protein Atlas ${ }^{44}$ human PIH1D3 is expressed in ciliated tissues, being highest in lung, fallopian tube and testis, supporting our evidence that PIH1D3 function in humans, unlike in mice, is not split between two isoforms.

Several other lines of evidence in this study reveal the possible biological role for PIH1D3 in cilia and flagella assembly. PIH1D3 expression is upregulated in parallel with other PCD genes during human respiratory epithelial ciliogenesis. PIH1D3 is exclusively cytoplasmic in multiciliated respiratory epithelial cells, a localization disrupted in PIH1D3-mutated cilia. PIH1D3 is highly conserved, as confirmed by recapitulation of structure and motility defects in PIH1D3-mutated human cilia and in hi1392Tg zebrafish pih1d3-null mutants, and the phenotypic rescue of 


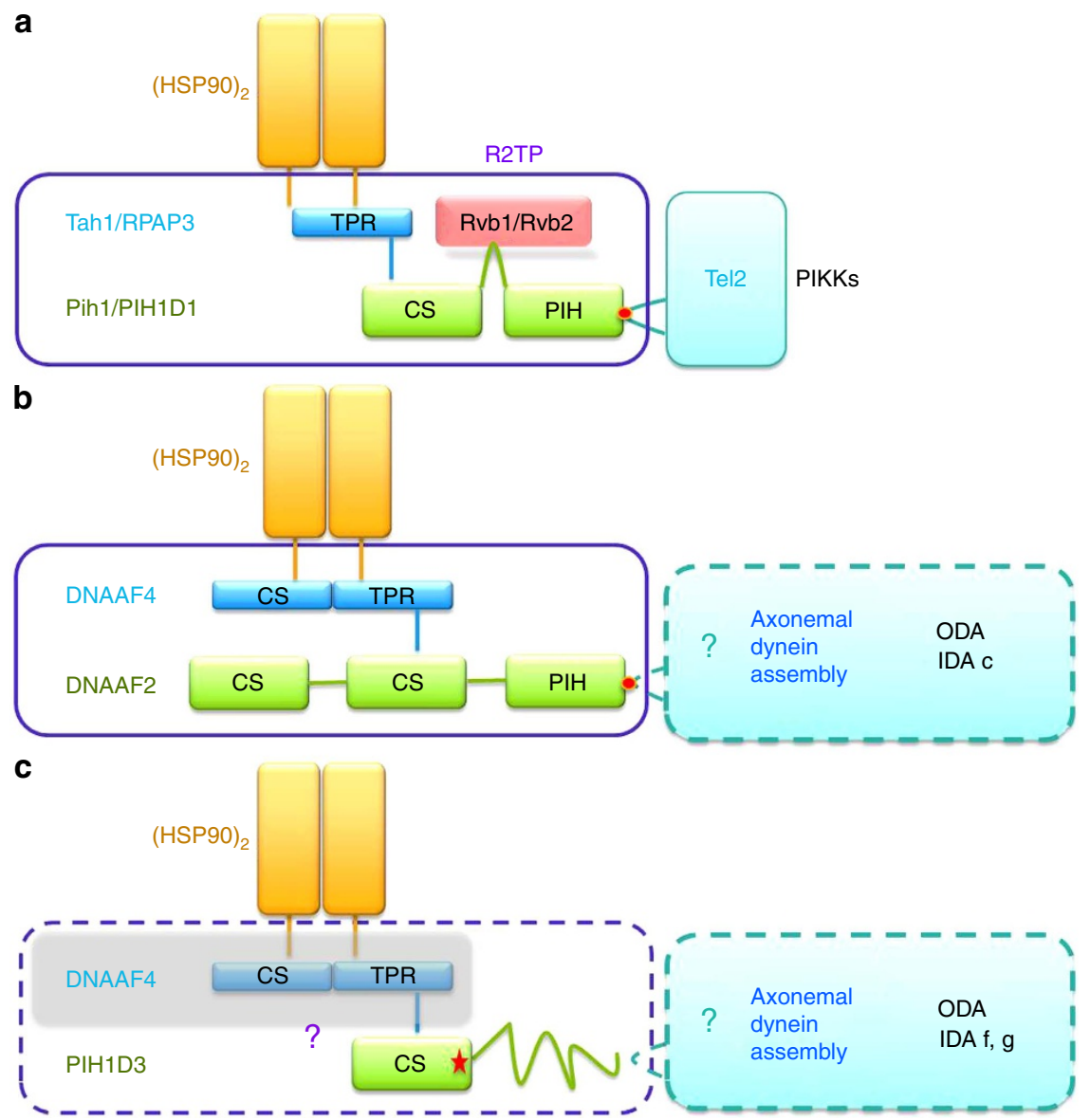

Figure 7 | Proposed model of PIH1D3 function in HSP90-mediated axonemal dynein assembly. PIH1D3 may participate in an R2TP-like co-chaperone complex composed of TPR (tetratricopeptide repeat), CS (CHORD-containing proteins and SGT1) and PIH1 (protein interacting with heat shock protein 90)-containing proteins to chaperone HSP9O for pre-assembly of axonemal dyneins in the cytoplasm, before their IFT-associated import into motile cilia and flagella. The proposed model compares PIH1D3 to two other cilia dysmotility-associated CS/PIH proteins mutated in PCD, DNAAF2 and DNAAF4 which are known to interact. (a) The yeast R2TP complex forms a multi-protein co-chaperone (purple box, human orthologs also shown) that recruits HSP90 dimer for activation of phosphoinositol-kinase like kinases (PIKKs) through a novel phosphorylation motif (red spot) in Tel2 that associates with the $\mathrm{PIH}$ domain of Pih1. (b) A structurally homologous co-chaperone DNAAF2/DNAAF4 module is proposed that may recruit HSP90 and HSP70 to axonemal dynein assembly. The phospho-motif binding site is conserved in the DNAAF2 PIH domain (red spot), although the phosphorylated partner is unknown. (c) CS domain similarity between PIH1D3 and DNAAF2 shows PIH1D3 may participate in a distinct co-chaperone module also required for axonemal dynein assembly. PIH1D3 does not contain a PIH domain, therefore, a different motif may be used to link to other proteins within the dynein assembly pathwaythe unstructured $\mathrm{N}$ terminus of PIH1D3, shown as a ribbon, is predicted to become structured on binding of a protein partner. The PCD-associated mutation p.Asp133Tyr (red star) within the CS domain disrupts interaction between PIH1D3 and DNAI2, an early component of axonemal dynein assembly. It is plausible that like DNAAF2, PIH1D3 could serve its function by an interaction with DNAAF4 (grey box). Biochemical analysis in Chlamydomonas and tomography data presented here suggest DNAAF2 and PIH1D3 are both involved in ODA assembly, and also in assembly of specific IDA dyneins. Boxes with solid lines represent known complexes and boxes with dashed lines possible complexes. ODA, outer arm dyneins; IDA, inner arm dyneins.

zebrafish mutants by human PIH1D3. Affected boys and the zebrafish model both show that PIH1D3 mutations result in loss of ODAs and IDAs. Ultrastructural studies detected some variation in the degree of ODA versus IDA loss in different patients carrying PIH1D3 mutations, which might be explained by their different genetic backgrounds or by different severity of specific mutations. Its cytoplasm localization and link to loss of dynein arms indicate that PIH1D3 is part of the same pathway as axonemal dynein assembly factors including DNAAF2-DNAAF4 that are likely co-chaperones of HSP90 (refs 25,26). Mutations in DNAAF2 and DNAAF4 also cause PCD with joint ODA and IDA loss. We have shown that like DNAAF2, PIH1D3 interacts in vitro with DNAI2, an intermediate dynein representing one of the first components of the human ODA assembly process.

Clinical tomography imaging of the entire IDA shows both complete or partial loss of IDA dyneins in PIH1D3-mutant cilia.
Little is known about human IDA assembly but similarly to Chlamydomonas, the human IDA contains seven major species of dyneins labelled $a-g^{45,46}$. We find in PIH1D3-mutant cilia where there is a partially assembled IDA, that the IDA dynein subunits $f$ and g specifically fail to assemble. Selective assembly of different IDA dynein subunits by different assembly factors was previously hypothesized in Chlamydomonas ${ }^{22}$. Our data indicating that specific assembly of dynein $\mathrm{f}$ and $\mathrm{g}$ (but not a or $\mathrm{c}$ ) is under the control of PIH1D3, possibly in a complementary manner to IDA dynein assembly by DNAAF2, supports a differentiation in the pathways of human axonemal dynein assembly, whereby different assembly factors assemble different subunits and PIH1D3 works alongside DNAAF2 to assemble complementary sets of IDA dyneins.

Our combined structural and functional data have highlighted a ciliary role for PIH1D3 in assembly of both the IDAs and 
ODAs. Recent analysis suggesting two known assembly factors DNAAF2 and DNAAF4 might form an R2TP-like complex for dynein assembly ${ }^{26}$ helps further define the possible function of PIH1D3 within this pathway (Fig. 7). First described in yeast, the R2TP co-chaperone regulates essential HSP90-related functions to direct the assembly of many multi-subunit complexes, including the phosphatidylinositol-3-kinase-like kinases (PIKKs) for signal transduction ${ }^{23}$. HSP90 is a molecular chaperone that folds and activates several hundred client proteins involved in many cellular processes, and its specificity of action is determined by numerous co-chaperones that regulate its ATPase activity and recruit the proteins it is required to fold ${ }^{47,48}$. In human R2TP, PIH1D1 acts as an adaptor linking HSP90 and RPAP3 (Tah1 in yeast) to RUVBL1 and RUVBL2 (yeast Rvb1/Rvb2) (Fig. 7a) ${ }^{23}$. The PIH domain of yeast Pih1 (human PIH1D1) was recently resolved as a phosphopeptide-binding domain that associates with a novel phosphorylation motif (DpSDDxF) in Tel2 to regulate PIKKs (Fig. 7a) ${ }^{26}$. Recruitment of R2TP to HSP90 is mediated through the $\mathrm{C}$ terminus CS domain of Pih1/PIH1D1 by TPR protein Tah1/RPAP3 that binds a conserved motif (MEEVD) of HSP90.

CS/TPR-containing DNAAF4 and CS/PIH-containing DNAAF2 are similar to the TPR domain protein RPAP3 and the CS/PIH protein PIH1D1, and evidence that the two interact with each other and with HSP90 and HSP70 (refs 9,24) suggests they could form an R2TP-like co-chaperone complex participating in axonemal dynein assembly ${ }^{25,26}$ (Fig. 7b). The phosphomotif binding site identified in Pihl/PIH1D1 (ref. 26) is conserved in the DNAAF2 PIH domain, indicating a similar function in mediating phosphorylation-dependent proteinprotein interactions, though the phosphorylated partner is unknown (Fig. 7b, red dot). The interaction of DNAAF2 with DNAI2 could indicate its link into the dynein assembly pathway (Fig. 7b). In the case of PIH1D3 (Fig. 7c), sequence and structural homology between its CS domain and that in DNAAF2 suggests that it could also interact with HSP90 though a TPR-containing intermediary (possibly but not necessarily DNAAF4), thereby acting as a co-chaperone recruiting HSP90 chaperone function into axonemal dynein assembly. The p.Asp133Tyr missense change that disrupts PIH1D3 interaction with DNAI2 confirms the functional importance of this residue within the PIH1D3 CS domain that might link directly to dynein assembly or indirectly via another assembly factor (Fig. 7c, red star).

By inference to the mouse testis-specific PIH1D3 homologue ${ }^{30}$, it is possible that human PIH1D3 is not associated with DNAAF1, DNAAF2 and DNAAF3, and that it functions separately during the assembly of dynein arm complexes. We find PIH1D3 is essential for assembly of the ODAs, and for IDA assembly it appears more strongly associated with assembly of only specific IDA subunits, the IDA dyneins $\mathrm{f}$ and $\mathrm{g}$ (Fig. 7c). Chlamydomonas biochemistry has previously suggested that DNAAF2 specifically assembles ODAs and IDA dynein subunit c (Fig. 7b) ${ }^{7}$. Our data appear to confirm some complementarity amongst human assembly factors as well, since isolated loss of dynein $\mathrm{c}$ was never seen in the PIH1D3-mutant cilia of PCD12 II:1 (Fig. 6). The remaining human IDA dyneins could be assembled specifically by other assembly factors and no human ortholog has yet reliably been identified for MOT48, which is a third PIH-domain cytoplasmic dynein assembly factor of Chlamydomonas that appears to assemble its other IDA dyneins, although MOT48 overlaps somewhat with DNAAF2 since it is thought to specifically assemble IDA dyneins b, c, d and $\mathrm{e}^{22,30}$.

In conclusion, we propose that PIH1D3 is a cytoplasmic protein involved in early axonemal dynein arm assembly as a likely HSP90 co-chaperone participating to assemble ODAs and certain IDA dynein subunits. PIH1D3 mutations affect its interaction with protein partner(s) in dynein assembly and give rise to a classic form of PCD disease with dynein arm loss. PIH1D3, therefore, joins the family of human cytoplasmic $\mathrm{PIH} / \mathrm{CS}$ dynein assembly factors, probably acting in a complex in a similar manner to the putative DNAAF2-DNAAF4 co-chaperone. We propose that it could be re-named DNAAF6 to prevent confusion about its structural domains. Better understanding of dynein assembly factors and their chaperonemediated interactions in PCD may provide an important target for therapy in future. Our findings of X-linked PCD have clinical importance for improved counselling of individuals affected by PCD and their families, in what has up until now been described as a largely autosomal recessive condition. PIH1D3 should be prioritized for mutational screening in PCD cases lacking dynein arms and in cases of PCD with a suggestive X-linked inheritance pattern.

\section{Methods}

Patients and sample collection. Individuals included in the study had a clinical diagnosis of PCD confirmed by standard clinical diagnostic criteria documenting typical symptoms of neonatal respiratory distress and chronic respiratory disease features including rhinosinusitis, re-occurring airway infections and productive cough, otitis media and bronchiectasis. Clinical test results included nasal nitric oxide measurements, medical imaging, in addition to light, electron and immunofluorescence microscopy to detect ciliary motility and analyse ciliary structure. For studies of affected individuals and their families, signed and informed consent was obtained from all participants before history recording, blood drawing and nasal biopsy, using protocols approved by the ethics review boards of the University Medical School in Geneva (Switzerland), the Institute of Child Health/Great Ormond Street Hospital, London (UK) (\#08/H0713/82) and the Comité de Protection des Personnes CPP Ile-de-France III (France) (approvals no. CPP07729 and CPP02748).

Next-generation sequence analysis. DNA was extracted from whole blood of affected individuals and family members by standard methods, with automated platforms such as Qiagen Autopure LS and NucliSENS used for the Geneva cohort. In the UK, the PCD12 sample was included in a cohort of 43 affected individuals subject to whole-exome sequencing as part of the UK10K project ${ }^{49}$. PCD392 was one of 48 affected individuals screened by targeted sequence capture. This used the SureSelectXT system (Agilent) and sequence capture libraries designed using the Agilent SureDesign wizard, to capture the full transcript of 640 target genes plus 25 basepairs (bp) at each intron-exon boundaries. A total of $25 \mathrm{ng}$ of genomic DNA was fragmented with QXT enzyme and tagged with adaptors for initial amplification. Libraries were captured and sequencing performed using standard protocols for the Illumina HiSeq platform (paired ends, $2 \times 150 \mathrm{bp}$ ). Barcoding allowed multiplexing of eight cases per flow cell. Sequence data was analysed using an in-house pipeline ${ }^{50}$ with sequence reads in FASTQ format aligned to the reference human genome (hg19) using BWA (0.6.1-r104) and default settings ${ }^{51}$. Variant calling was performed across the entire region of interest using VarScan2 (V.2.3.7) set at minimum $30 \times$ coverage, minimum five alternate reads, minimum phred-like base-quality of 20 (ref. 52). Variant calls in VCF format were then annotated using Ensembl Variant Effect Predictor (V.73) and the output parsed using an in-house script, which converts the annotated VCF file into Excel format for subsequent variant filtering and prioritization ${ }^{53}$. In Geneva, genomic DNA of 59 affected individuals (belonging to 47 families) was fragmented by sonication to $200 \mathrm{bp}$ fragments then liquid phase captured against whole human exome probes to select all coding sequences (SureselectXT Target Enrichment System Version 1.2, Agilent). Sequencing was performed on an Illumina HiSeq 2000 instrument (paired ends, $2 \times 95 \mathrm{bp}$ ). Each exome library was indexed, separated into two equal halves and sequenced in two different lanes, up to five half-libraries were sequenced in each HiSeq lane. The raw results were analysed using a local bioinformatics pipeline, which as previously described ${ }^{16}$ combines published algorithms in a sequential manner (BWA for map reads, SAMtool for detection of variants, Pindel for the detection of indels, ANNOVAR for annotation of sequence changes). The entire coding sequence corresponding to RefSeq

(http://www.ncbi.nlm.nih.gov/refseq/) coding genes was used as the reference for the calculation of coverage and reads on target.

In both centres, for subsequent filtering of variants, only exonic and splicing variants ( $\pm 10 \mathrm{bp}$ of the intron-exon boundary) were retained for further analysis These were filtered to exclude synonymous variants, variants with a minimum allele frequency $\geq 0.01$ in dbSNP version 137, 1000Genomes (http:// 1000 genomes.org/), local databases and variants found within segmental duplications of the genome. Minor allele frequency was then checked on Exome Variant Server (http://evs.gs.washington.edu/EVS/) and ExAC (http:// exac.broadinstitute.org/) databases, as well as in VariantMaster ${ }^{54}$ in Geneva, to further filter for variants common among the affected individuals. Final filtered 
variants were evaluated individually based on the predicted pathogenicity scores provided by a number of softwares including SIFT, PolyPhen-2 and Mutation Taster, their presence in HGMD (http://www.hgmd.cf.ac.uk/ac/index.php) and the literature, focusing on functional data. Evolutionary conservation scores were provided by PhyloP and GERP ++ .

Whole-genome SNP-array analysis. Patients were genotyped for copy number variations with the HumanOmniExpress 24 BeadChip from Illumina and the data were analysed with the Genome Studio and CNV partition 3.1.6 softwares (Illumina).

Sanger sequencing. Sanger sequencing of custom-designed PIH1D3 amplicons (NM_001169154) confirmed potentially disease-causing variants in probands and allowed segregation analysis in their families. Primers were designed using Primer 3 (http://primer3.ut.ee/) and are listed in Supplementary Table 3 with PCR conditions available on request. Sequences were analysed and compared with controls using Sequencher software (Gene Codes Corporation, Ann Arbor, MI, USA) in UK patients, Mutation Surveyor (SoftGenetics, LLC) and Staden (http:// staden.sourceforge.net/) in the Geneva patients and Seqscape software (Applied Biosystems, Foster City, USA) in the French patients.

Immunofluorescence analysis. All antibodies used are listed in Supplementary Tables 4 and 5. Respiratory epithelial cells obtained by nasal brush biopsy and suspended in cell culture media were air dried onto glass slides and stored at $-80^{\circ} \mathrm{C}$ until use. Immunostaining on the slides was performed as described ${ }^{55}$. The cells were incubated with primary antibodies overnight at room temperature at the dilutions listed in Supplementary Table 4, then with secondary antibodies diluted at 1:1,000 dilution for an hour. Cells were finally washed five times with PBS, mounted in ProLong Gold Antifade Mountant with DAPI (ThermoFisher Scientific) and confocal images were taken using a Zeiss LSM 710 (Zeiss, Cambridge, UK). Immunofluoresence analysis in Supplementary Fig. 3 verified a Sigma PIH1D3 antibody by specific detection of PIH1D3 at the expected molecular weight.

cDNA cloning and site-directed mutagenesis. Fully sequenced human PIH1D3 (clone 5173807; Genbank NM_001169154) and DNAI2 (clone 5752153; Genbank NM_023036) cDNA clones were purchased from Mammalian Gene Collection (GE Healthcare) for Gateway Cloning. PCR products were amplified using PWO high-fidelity Taq Polymerse (Roche), subcloned into Gateway entry vector (ThermoFisher) and recombined with pcDNA-DEST53 (ThermoFisher) and pCMV-Myc (Clontech) Gateway Vector via LR Clonase II encoding GFP and Myc $\mathrm{N}$-terminal epitope tags, respectively.

Primers for mutagenesis were designed according to the QuikChange Primer Design Tool (Agilent Technologies). Tagged plasmids were amplified using KAPA HiFi DNA Polymerase (KAPA Biosystems) according to the manufacturer's directions. Amplified products were gel purified and Dpn1 digested for $2 \mathrm{~h}$ before transforming into competent XL1-Blue cells (Stratagene). All cDNA and mutant clones were verified by Sanger sequencing. cDNA cloning and site-directed mutagenesis primers are listed in Supplementary Table 3.

Cell culture and transfection. Human embryonic kidney cells (HEK293T, American Type Culture Collection) were grown to confluence in DMEM (Life Technologies) supplemented with 10\% fetal bovine serum and $1 \%$ penicillinstreptomycin antibiotic (Invitrogen). Cultures were plated at a density of $6 \times 10^{5}$ cells per well in a 6-well plate. Cells were transfected with plasmids encoding GFP-tagged cDNA constructs, or both Myc- and GFP- tagged plasmids were co-expressed for co-IP assays. DNA $(2 \mu \mathrm{g})$ was added to each well with $8 \mu \mathrm{l}$ of Lipofectamine 2000 reagent (Thermo Scientific) in OptiMEM-1 (Invitrogen) and $4 \mu \mathrm{g}$ DNA (each at $2 \mu \mathrm{g}$ ) for co-expression studies. Within $48 \mathrm{~h}$, cells were collected in $1 \times$ PBS and lysed in $300 \mathrm{ul}$ of $20 \mathrm{mM}$ Tris-Cl (pH 7.4), $50 \mathrm{mM} \mathrm{NaCl}$ and $1 \%$ NP40 supplemented with protease inhibitor cocktail (Sigma). Lysates were centrifuged at $16,000 \mathrm{~g}$ for $15 \mathrm{~min}$ at $4{ }^{\circ} \mathrm{C}$, aliquoted and stored at $-20^{\circ} \mathrm{C}$ until use.

Co-IP and immunoblotting. IP assays were performed using Dynabeads Protein G beads (Novex). Briefly, $20 \mu \mathrm{l}$ of Dynabeads were washed three times in IP buffer (50 mM Tris 7.4, $150 \mathrm{mM} \mathrm{NaCl}, 1 \mathrm{mM}$ EDTA, $0.5 \%$ sodium deoxycholate and $1 \%$ NP40). Approximately $2 \mathrm{mg}$ of co-expressed lysate with protease inhibitor cocktail (Sigma) was added to pre-washed beads to pre-clear on a rotator for $2 \mathrm{~h}$ at $4{ }^{\circ} \mathrm{C}$. Subsequently, $50 \mu$ l of beads were briefly washed three times in IP buffer and then incubated with $1 \mu \mathrm{g}$ of mouse IgG anti-GFP (clones 7.1 and 13.1, Roche) antibody at room temperature for $1.5 \mathrm{~h}$. Antibody-bead complexes were gently washed three times in IP buffer before incubating with pre-cleared lysate overnight at $4{ }^{\circ} \mathrm{C}$. Bead complexes were gently washed five times in IP buffer and resuspended in $2 \times$ Laemmli sample buffer (BioRad), $1 \mu \mathrm{l}$ of 2-mercaptoethanol (Sigma) and heated for $10 \mathrm{~min}$ at $95^{\circ} \mathrm{C}$ to dissociate the complex from the beads. Beads were separated on a magnet and lysates were then by electrophoresed on 10\% SDS-PAGE gels, transferred to nitrocellulose membranes and blocked in 5\% skimmed milk, 3\% BSA dissolved in PBS-Tween for $2 \mathrm{~h}$ at room temperature before immunoblotting overnight at $4{ }^{\circ} \mathrm{C}$ with mouse anti-Myc (9E10, ThermoFisher). For immunoblotting, $20 \mu \mathrm{g}$ of lysate was resuspended in $2 \times$ Laemmli sample buffer (BioRad), $1 \mu$ l of 2-mercaptoethanol (Sigma) and heated for $5 \mathrm{~min}$ at $95^{\circ} \mathrm{C}$ and the protocol continued similarly to the IP assay. Membranes were washed four times (10 min each) then incubated with anti-mouse HRP or anti-sheep HRP secondary antibody for $45 \mathrm{~min}$ at room temperature. Membranes were then washed five times and developed by Pierce ECL Western Blotting Substrate (ThermoFisher Scientific). Images were developed on X-ray films (GE Healthcare).

The following antibodies were used: mouse monoclonal anti-GFP (clones 7.1 and 13.1, Roche), mouse monoclonal anti-Myc (1:1,000, clone 9E10, Merck Millipore), rabbit polyclonal anti-PIH1D3 (1:400, HPA051099, Sigma), mouse anti-GAPDH (1:1,500, clone 6C5, Merck Millipore), donkey anti-rabbit HRP antibody (1:5,000, NA934, GE Healthcare) and sheep anti-mouse HRP antibody (1:5,000, NXA931, GE Healthcare).

Air-liquid interface (ALI) cultures. Normal human bronchial epithelial (NHBE) cells were obtained from Lonza. Cultures were transformed at passage 2 with Bmil and maintained on collagen-coated plates with Bronchial Epithelial Growth Media (Lonza) for 7 days to grow to confluence. Cells were seeded at a density of $5 \times 10^{5}$ onto 24-well collagen-coated transwell inserts (Costar, Corning, USA). When confluent after $48 \mathrm{hr}$ cell the cultures were exposed to an air-liquid interface (ALI) as previously described ${ }^{56}$ by removing medium from their surface (apical chamber) and replacing the medium in the basal chamber with ALI medium (1:1 ratio of DMEM $4.5 \mathrm{gl}^{-1}$ D-glucose:Bronchial Epithelial Growth Media containing $100 \mathrm{nM}$ all-trans retinoic acid (Sigma-Aldrich, UK)). Cells were ALI cultured for the next 30 days.

Quantitative RT-PCR analysis. Total RNA was isolated from ALI culture pellets using Trizol Reagent (Invitrogen) and used to produce complementary DNA with Omniscript (Invitrogen). RT-qPCR primers were designed using Primer-BLAST (http://www.ncbi.nlm.nih.gov/tools/primer-blast/) to ensure specificity and primer sets were optimized across a range of cDNA concentrations by generating standard and melt curves per gene. All real-time qPCR reactions were carried out with a CFX96 Touch Real-Time PCR Detection instrument (Biorad) with samples set up in triplicates in $20 \mu \mathrm{l}$ reactions containing $2 \times$ iQ SYBR Green Supermix (Biorad), 3 picomolar of $100 \mu \mathrm{M}$ primer mix (forward and reverse primers) and $500 \mathrm{ng}$ of cDNA. GAPDH was used as an endogenous control for normalization between target gene expression levels. Data acquisition of the fluorescent signal was performed at the end of the run. Double delta CT calculations were measured as logarithm and then converted to fold change of 100, after untreated control levels were subtracted from target levels. Primers used in RT-qPCR experiments are listed in Supplementary Table 3.

Zebrafish analysis. All zebrafish (Danio rerio) experiments were approved by the Institutional Animal Care and Use Committee (IACUC) of Yale University. Husbandry was carried out according to standard protocols ${ }^{57}$. A human rescue expression vector was created by cloning human PIH1D3 (PCR primers $5^{\prime}$-CACCAGGATCCATGGAATCTGAAAATATGGAT- ${ }^{\prime}$ and $5^{\prime}$-AGGCTCGAG TCAGAAGAAATTAGCAATATC- $3^{\prime}$ ) into the pCS2 + vector between BamHI and XhoI restriction endonuclease sites. For zebrafish pihld3, total RNA was isolated from wild-type TuAB embryos, followed by cDNA synthesis with High-Capacity cDNA Reverse Transcription Kit (Applied Biosystems). PCR products using pihld 3 -specific primers $5^{\prime}$-(ggatcccatcgattcg)ATGGAGGGTCTCG CGTCC- $3^{\prime}$ and $5^{\prime}$-(actatagttctagaggc)TCAGGTCAGATTAATGCAGTCCA- $3^{\prime}$ were cloned using the Gibson assembly kit (NEB) into an EcoRI- and XhoIdigested pCS2 + vector. Plasmids were linearized with NotI, followed by $5^{\prime}$ cap mRNA synthesis using the SP6 mMESSAGE mMACHINE kit (Ambion). Purified mRNA was injected at $1 \mathrm{nl}$ into 1-cell stage embryos. The embryos were raised at $+28.5^{\circ} \mathrm{C}$ until analysis. For genotyping, embryos were lysed with $50 \mathrm{mM} \mathrm{NaOH}$, and analysed by duplex PCR using primers $5^{\prime}$-TTACTACTACAATCCG CGTCCATG- ${ }^{\prime}, 5^{\prime}$-CAAGGTTTGTCAGGCGATAATAGAT- $3^{\prime}$ and $5^{\prime}$-GCTAG CTTGCCAAACCTACAGGT-3'. For mRNA expression, mRNA was purified using Trizol (Invitrogen), followed by cDNA synthesis using the High-Capacity cDNA Reverse Transcription Kit (Applied Biosystems) and PCR with primers against full-length pihld3, $5^{\prime}$-ATGGAGGGTCTCGCGTCC- $3^{\prime}$ and $5^{\prime}$-TCAGGTC AGATTAATGCAGTCCATTG-3'. For histology, 50 hpf embryos were anesthetized and fixed with Bouin's fixative overnight at room temperature. Fixed embryos were washed with PBT (PBS and 0.1\% (v/v) Tween 20), dehydrated and rehydrated with $100 \%$ methanol, washed with PBT and embedded in JB-4 plastic resin (Polysciences). The moulds were cut into $4 \mu \mathrm{m}$ slices and stained with hematoxylin and eosin. For TEM of zebrafish, embryos were anesthetized and fixed in $3 \%$ glutaraldehyde and $1.5 \%$ paraformaldehyde in a $0.1 \mathrm{M}$ phosphate buffer $(\mathrm{pH}$ 7.4) and subsequently stored in $0.1 \mathrm{M}$ phosphate buffer supplemented with $0.01 \%$ sodium azide for transportation. Before TEM, embryos were washed overnight and post-fixed in $1 \%$ osmium tetroxide. After dehydration, the samples were embedded in epoxy resin. Thin sections stained with Reynold's lead citrate were imaged with a Jeol 1010 electron microscope. For high-speed microscopy and analysis in zebrafish, embryos were anesthetized with $0.2 \mathrm{ml} \mathrm{ml}^{-1}$ Tricaine (Sigma-Aldrich) and mounted on microscope slides in embryo medium. DIC images were acquired through a $\times 40$ water immersion objective on a Zeiss Observer z1 fitted with a 
Hamamatsu Orca Flash 4.0 V2 high-speed camera. Kymographs were created from 0.5 -second 1,000 fps videos using ImageJ software.

Electron tomography. Nasal brushings were chemically fixed and embedded as previously described ${ }^{58}$. In brief; biopsies were obtained from the inferior turbinate of the nose using a cytology brush. For electron microscopy, samples were fixed in $2.5 \%$ glutaraldehyde, washed with cacodylate buffer, post-fixed in osmium tetroxide and rinsed with distilled water, with centrifuging taking place between each step. Epithelial strips were bound with $2 \%$ agar and then dehydrated and infiltrated with araldite resin and embedded in araldite. Ciliated areas were selected in survey sections before cutting dark gold-blue ultra thin sections. Ultra thin sections $(\sim 150 \mathrm{~nm})$ were stained with methanolic uranyl acetate and lead citrate and $10 \mathrm{~nm}$ gold fidicuals were added to aid correlation of the captured images for tomogram generation.

Images of cilia in longitudinal section were acquired at $\times 20,000$ magnification using a Jeol $1400+$ transmission electron microscope with a mid-mount digital camera (AMT16X). Automated Recorder software package v2.7 (Jeol Technology Systems) was used to acquire dual axis tilt sequences between $70^{\circ}$ and $-70^{\circ}$ in $1^{\circ}$ increments. Tomograms were then generated using IMOD software created by the University of Colorado ${ }^{59,60}$. Particle Estimation for Electron Tomography (PEET) software was used to average and align particles along manually selected microtubules ${ }^{61}$. The outcome of the PEET process is a density map, representing an average $96 \mathrm{~nm}$ repeat of the selected tubule in which regular, repeated features are emphasized. The density maps were opened within the UCSF Chimera graphics package ${ }^{62}$. A data threshold was set for each individual map based on achieving a balance between structural detail and background noise. The Segger tool was used to split the volume map into distinct segments which were then isolated and coloured to aid visualization.

Data availability. All the relevant data are available from the authors upon request.

\section{References}

1. Ishikawa, T. Axoneme structure from motile cilia. Cold Spring Harb Perspect Biol. doi: 10.1101/cshperspect.a028076 (2016).

2. Lucas, J. S. et al. Diagnosis and management of primary ciliary dyskinesia. Arch. Dis. Child. 99, 850-856 (2014).

3. Boon, M. et al. MCIDAS mutations result in a mucociliary clearance disorder with reduced generation of multiple motile cilia. Nat. Commun. 5, 4418 (2014).

4. Shapiro, A. J. et al. Laterality defects other than situs inversus totalis in primary ciliary dyskinesia: insights into situs ambiguus and heterotaxy. Chest 146, 1176-1186 (2014)

5. Duquesnoy, P. et al. Loss-of-function mutations in the human ortholog of Chlamydomonas reinhardtii ODA7 disrupt dynein arm assembly and cause primary ciliary dyskinesia. Am. J. Hum. Genet. 85, 890-896 (2009).

6. Loges, N. T. et al. Deletions and point mutations of LRRC50 cause primary ciliary dyskinesia due to dynein arm defects. Am. J. Hum. Genet. 85, 883-889 (2009).

7. Omran, H. et al. Ktu/PF13 is required for cytoplasmic pre-assembly of axonemal dyneins. Nature 456, 611-616 (2008).

8. Mitchison, H. M. et al. Mutations in axonemal dynein assembly factor DNAAF3 cause primary ciliary dyskinesia. Nat. Genet. 44, 381-389 (2012).

9. Tarkar, A. et al. DYX1C1 is required for axonemal dynein assembly and ciliary motility. Nat. Genet. 45, 995-1003 (2013).

10. Horani, A. et al. Whole-exome capture and sequencing identifies HEATR2 mutation as a cause of primary ciliary dyskinesia. Am. J. Hum. Genet. 91, 685-693 (2012).

11. Diggle, C. P. et al. HEATR2 plays a conserved role in assembly of the ciliary motile apparatus. PLoS Genet. 10, e1004577 (2014).

12. Knowles, M. R. et al. Mutations in SPAG1 cause primary ciliary dyskinesia associated with defective outer and inner dynein arms. Am. J. Hum. Genet. 93, 711-720 (2013).

13. Austin-Tse, C. et al. Zebrafish ciliopathy screen plus human mutational analysis identifies C21orf59 and CCDC65 defects as causing primary ciliary dyskinesia. Am. J. Hum. Genet. 93, 672-686 (2013).

14. Kott, E. et al. Loss-of-function mutations in LRRC6, a gene essential for proper axonemal assembly of inner and outer dynein arms, cause primary ciliary dyskinesia. Am. J. Hum. Genet. 91, 958-964 (2012).

15. Horani, A. et al. LRRC6 mutation causes primary ciliary dyskinesia with dynein arm defects. PLoS ONE 8, e59436 (2013).

16. Moore, D. J. et al. Mutations in ZMYND10, a gene essential for proper axonemal assembly of inner and outer dynein arms in humans and flies, cause primary ciliary dyskinesia. Am. J. Hum. Genet. 93, 346-356 (2013).

17. Zariwala, M. A. et al. ZMYND10 is mutated in primary ciliary dyskinesia and interacts with LRRC6. Am. J. Hum. Genet. 93, 336-345 (2013).

18. King, S. M. \& Patel-King, R. S. The oligomeric outer dynein arm assembly factor CCDC103 is tightly integrated within the ciliary axoneme and exhibits periodic binding to microtubules. J. Biol. Chem. 290, 7388-7401 (2015).
19. Panizzi, J. R. et al. CCDC103 mutations cause primary ciliary dyskinesia by disrupting assembly of ciliary dynein arms. Nat. Genet. 44, 714-719 (2012).

20. Kobayashi, D. \& Takeda, H. Ciliary motility: the components and cytoplasmic preassembly mechanisms of the axonemal dyneins. Differentiation 83, S23-S29 (2012).

21. Rosenbaum, J. L. \& Witman, G. B. Intraflagellar transport. Nat. Rev. Mol. Cell Biol. 3, 813-825 (2002).

22. Yamamoto, R., Hirono, M. \& Kamiya, R. Discrete PIH proteins function in the cytoplasmic preassembly of different subsets of axonemal dyneins. J. Cell Biol. 190, 65-71 (2010).

23. Kakihara, Y. \& Houry, W. A. The R2TP complex: discovery and functions. Biochim. Biophys. Acta 1823, 101-107 (2012).

24. Chen, Y. et al. A novel role for DYX1C1, a chaperone protein for both Hsp70 and Hsp90, in breast cancer. J. Cancer Res. Clin. Oncol. 135, 1265-1276 (2009).

25. Vaughan, C. K. Hsp90 picks PIKKs via R2TP and Tel2. Structure 22, 799-800 (2014).

26. Pal, M. et al. Structural basis for phosphorylation-dependent recruitment of Tel2 to Hsp90 by Pih1. Structure 22, 805-818 (2014).

27. Jaffe, K. M. et al. c21orf59/kurly controls both cilia motility and polarization. Cell Rep. 14, 1841-1849 (2016).

28. Wu, J., Luo, S., Jiang, H. \& Li, H. Mammalian CHORD-containing protein 1 is a novel heat shock protein 90-interacting protein. FEBS Lett. 579, 421-426 (2005).

29. Garcia-Ranea, J. A., Mirey, G., Camonis, J. \& Valencia, A. p23 and HSP20/alpha-crystallin proteins define a conserved sequence domain present in other eukaryotic protein families. FEBS Lett. 529, 162-167 (2002).

30. Dong, F. et al. Pih1d3 is required for cytoplasmic preassembly of axonemal dynein in mouse sperm. J. Cell Biol. 204, 203-213 (2014).

31. Yeo, G. \& Burge, C. B. Maximum entropy modeling of short sequence motifs with applications to RNA splicing signals. J. Comput. Biol. 11, 377-394 (2004)

32. Salamov, A. A., Nishikawa, T. \& Swindells, M. B. Assessing protein coding region integrity in cDNA sequencing projects. Bioinformatics 14, 384-390 (1998).

33. Kamiya, R. Functional diversity of axonemal dyneins as studied in Chlamydomonas mutants. Int. Rev. Cytol. 219, 115-155 (2002).

34. Chilvers, M. A., Rutman, A. \& O'Callaghan, C. Ciliary beat pattern is associated with specific ultrastructural defects in primary ciliary dyskinesia. J. Allergy Clin. Immunol. 112, 518-524 (2003).

35. Sun, Z. et al. A genetic screen in zebrafish identifies cilia genes as a principal cause of cystic kidney. Development 131, 4085-4093 (2004).

36. Kramer-Zucker, A. G. et al. Cilia-driven fluid flow in the zebrafish pronephros, brain and Kupffer's vesicle is required for normal organogenesis. Development 132, 1907-1921 (2005)

37. Essner, J. J., Amack, J. D., Nyholm, M. K., Harris, E. B. \& Yost, H. J. Kupffer's vesicle is a ciliated organ of asymmetry in the zebrafish embryo that initiates left-right development of the brain, heart and gut. Development 132, 1247-1260 (2005).

38. Lundberg, Y. W., Xu, Y., Thiessen, K. D. \& Kramer, K. L. Mechanisms of otoconia and otolith development. Dev. Dyn. 244, 239-253 (2015).

39. Fowkes, M. E. \& Mitchell, D. R. The role of preassembled cytoplasmic complexes in assembly of flagellar dynein subunits. Mol. Biol. Cell 9, 2337-2347 (1998).

40. Adzhubei, I. A. et al. A method and server for predicting damaging missense mutations. Nat. Methods 7, 248-249 (2010).

41. Kumar, P., Henikoff, S. \& Ng, P. C. Predicting the effects of coding nonsynonymous variants on protein function using the SIFT algorithm. Nat. Protoc. 4, 1073-1081 (2009).

42. Narayan, D. et al. Unusual inheritance of primary ciliary dyskinesia (Kartagener's syndrome). J. Med. Genet. 31, 493-496 (1994).

43. Moore, A. et al. RPGR is mutated in patients with a complex X linked phenotype combining primary ciliary dyskinesia and retinitis pigmentosa. J. Med. Genet. 43, 326-333 (2006).

44. Uhlen, M. et al. A human protein atlas for normal and cancer tissues based on antibody proteomics. Mol. Cell Proteomics 4, 1920-1932 (2005).

45. Hom, E. F. et al. A unified taxonomy for ciliary dyneins. Cytoskeleton $\mathbf{6 8}$, 555-565 (2011).

46. Porter, M. E. \& Sale, W. S. The $9+2$ axoneme anchors multiple inner arm dyneins and a network of kinases and phosphatases that control motility. J. Cell Biol. 151, F37-F42 (2000).

47. Li, J. \& Buchner, J. Structure, function and regulation of the hsp90 machinery. Biomed. J. 36, 106-117 (2013).

48. von Morgen, P., Horejsi, Z. \& Macurek, L. Substrate recognition and function of the R2TP complex in response to cellular stress. Front. Genet. 6, 69 (2015).

49. Onoufriadis, A. et al. Combined exome and whole-genome sequencing identifies mutations in ARMC4 as a cause of primary ciliary dyskinesia with defects in the outer dynein arm. J. Med. Genet. 51, 61-67 (2014).

50. Trump, N. et al. Improving diagnosis and broadening the phenotypes in early-onset seizure and severe developmental delay disorders through gene panel analysis. J. Med. Genet. 53, 310-317 (2016).

51. Li, H. \& Durbin, R. Fast and accurate short read alignment with Burrows-Wheeler transform. Bioinformatics 25, 1754-1760 (2009). 
52. Koboldt, D. C. et al. VarScan 2: somatic mutation and copy number alteration discovery in cancer by exome sequencing. Genome Res. 22, 568-576 (2012).

53. McLaren, W. et al. Deriving the consequences of genomic variants with the Ensembl API and SNP Effect Predictor. Bioinformatics 26, 2069-2070 (2010).

54. Santoni, F. A. et al. Simultaneous identification and prioritization of variants in familial, de novo, and somatic genetic disorders with VariantMaster. Genome Res. 24, 349-355 (2014).

55. Onoufriadis, A. et al. Targeted NGS gene panel identifies mutations in RSPH1 causing primary ciliary dyskinesia and a common mechanism for ciliary central pair agenesis due to radial spoke defects. Hum. Mol. Genet. 23, 3362-3374 (2014).

56. Hirst, R. A., Rutman, A., Williams, G. \& O'Callaghan, C. Ciliated air-liquid cultures as an aid to diagnostic testing of primary ciliary dyskinesia. Chest $\mathbf{1 3 8}$, 1441-1447 (2010).

57. Westerfield, M. The Zebrafish Book. A Guide for the Laboratory Use of Zebrafish (Danio rerio) (Univ. of Oregon Press, 2000).

58. Shoemark, A., Dixon, M., Corrin, B. \& Dewar, A. Twenty-year review of quantitative transmission electron microscopy for the diagnosis of primary ciliary dyskinesia. J. Clin. Pathol. 65, 267-271 (2012).

59. Kremer, J. R., Mastronarde, D. N. \& McIntosh, J. R. Computer visualization of three-dimensional image data using IMOD. J. Struct. Biol. 116, 71-76 (1996).

60. Mastronarde, D. N. Dual-axis tomography: an approach with alignment methods that preserve resolution. J. Struct. Biol. 120, 343-352 (1997).

61. Nicastro, D. et al. The molecular architecture of axonemes revealed by cryoelectron tomography. Science 313, 944-948 (2006).

62. Pettersen, E. F. et al. UCSF chimera-a visualization system for exploratory research and analysis. J. Comput. Chem. 25, 1605-1612 (2004).

63. Kelley, L. A., Mezulis, S., Yates, C. M., Wass, M. N. \& Sternberg, M. J. The Phyre2 web portal for protein modeling, prediction and analysis. Nat. Protoc. 10, 845-858 (2015).

\section{Acknowledgements}

We are very grateful to the families with PCD who have participated in this study and to the UK PCD Family Support Group for their support. We thank Celia D. Delozier, R. Lazor and Jane Hurst for family recruitment and their present or past involvement in the project. We thank Andrew Rogers and Emily Frost from the Royal Brompton Hospital and Dale Moulding (UCL-GOS-ICH Confocal Microscopy Core Facility) and Kim Harrison-Lavoie (UCL) for experimental assistance. We thank Federico A. Santoni (University of Geneva School of Medicine and University Hospitals of Geneva) for bioinformatics expertise. We thank Shiaulou Yuan (Department of Pediatrics, YSM) for assistance with high-speed microscopy and SueAnn Mentone (Department of Cellular and Molecular Physiology, YSM) for advice regarding TEM. A.B. is an NIHR Senior Investigator and additionally was supported by the NIHR Respiratory Disease Biomedical Research Unit at the Royal Brompton and Harefield NHS Foundation Trust and Imperial College London. Work by A.S. is independent research funded by a postdoctoral research fellowship from the National Institute of Health Research and Health Education England, and the views expressed in this publication are those of the authors and not necessarily those of the NHS, the National Institute of Health Research or the Department of Health. Z.S. and S.K. are supported by NIH Grants R01HL125885-01A1 and R01 DK092808-01A1. J.-L.B.is supported by a grant from the Swiss National Science Foundation (\#32003B_135709). J.-L.B., L.B., C.O. and S.A. are supported by grants from the Milena Carvajal Pro-Kartagener Foundation of Geneva; J.-L.B. and C.O. were supported by a grant from the Ernst et Lucie Schmidheiny foundation of Geneva. S.E.A and J.-L.B are supported by funds from the University Hospitals of Geneva. A.O. is supported by the National Institute for Health Research (NIHR) Biomedical Research Centre at Guy's and St Thomas' NHS Foundation Trust and King's College London. S.A. is supported by grants from the Fondation pour la Recherche Médicale (DEQ20120323689) and the Legs Poix from the Chancellerie des Universités. This research was supported by the National Institute for Health Research Biomedical Research Centre at Great Ormond Street Hospital for Children NHS Foundation Trust and University College London. We acknowledge Wellcome Trust award WT091310 to $\mathrm{UK} 10 \mathrm{~K}$ and are grateful to the UK10K consortium (investigators listed at http:// www.uk10k.org/), in particular the Rare Diseases Group, for whole-exome sequencing of PCD patients. Please note that A.O. and H.M.M. are UK10K Rare members, but listed in the main author group. H.M.M. was supported by grants from Action Medical Research (GN2101), Newlife Foundation (10-11/15) and the Great Ormond Street Hospital Children's Charity.

\section{Author contributions}

Study concept and design was by: A.S., M.L., E.E., C.H., S.A., Z.S., L.B., J.-L.B and H.M.M. Genetic methods performed by C.O., M.P.P., M.L., H.J.W., J.H., A.Go., L.D., J.B., C.G., M.G., S.C., P.D., L.T., L.Jea., B.C., M.R.F., L.Jen., C.B., T.Cu., A.O., UK10K, S.E.A L.B. and H.M.M. Molecular cell biology performed by M.P.P, C.O. and M.M.M. Protein modelling by R.D.E and C.K.V, and development of HSP90 interaction model C.K.V. and H.M.M. Zebrafish studies by S.K. and Z.S. Clinical ultrastructural studies by A.S., M.D. and E.E.; and T.Ca. and A.S. performed all the electron tomography. A.Go., A.T., C.T.-R., J.-F.P., A.Ga., I.P., G.V., P.A., A.B., E.M.K.C., M.R.L., R.W., M.A., E.E., C.H. and L.B. provided clinical data. A.S., C.K.V., S.K., Z.S., C.O., L.B., J.-L.B., S.A. and H.M.M. wrote the manuscript. All authors approved the final version submitted for publication.

\section{Additional information}

Supplementary Information accompanies this paper at http://www.nature.com/ naturecommunications

Competing financial interests: The authors declare no competing financial interests.

Reprints and permission information is available online at http://npg.nature.com/ reprintsandpermissions/

How to cite this article: Olcese, C. et al. X-linked primary ciliary dyskinesia due to mutations in the cytoplasmic axonemal dynein assembly factor PIH1D3. Nat. Commun 8, 14279 doi: $10.1038 /$ ncomms14279 (2017)

Publisher's note: Springer Nature remains neutral with regard to jurisdictional claims in published maps and institutional affiliations.

This work is licensed under a Creative Commons Attribution 4.0 International License. The images or other third party material in this article are included in the article's Creative Commons license, unless indicated otherwise in the credit line; if the material is not included under the Creative Commons license, users will need to obtain permission from the license holder to reproduce the material. To view a copy of this license, visit http://creativecommons.org/licenses/by/4.0/

(C) The Author(s) 2017

\section{UK10K Rare Group}

Saeed Al-Turki ${ }^{28,29}$, Carl Anderson ${ }^{28}$, Dinu Antony ${ }^{3}$, Inês Barroso ${ }^{28}$, Philip L. Beales ${ }^{3}$, Jamie Bentham ${ }^{30}$, Shoumo Bhattacharya ${ }^{30}$, Keren Carss ${ }^{28}$, Krishna Chatterjee ${ }^{31}$, Sebahattin Cirak ${ }^{32}$, Catherine Cosgrove ${ }^{30}$, Daly Allan ${ }^{28}$, Richard Durbin ${ }^{28}$, David Fitzpatrick ${ }^{33}$, Jamie Floyd ${ }^{28}$, A. Reghan Foley ${ }^{32}$, Chris Franklin ${ }^{28,}$ Marta Futema ${ }^{34}$, Steve E. Humphries ${ }^{34}$, Matt Hurles ${ }^{28}$, Shane McCarthy ${ }^{28}$, Dawn Muddyman ${ }^{28}$, Francesco Muntoni ${ }^{32}$, Victoria Parker ${ }^{31}$, Felicity Payne ${ }^{28}$, Vincent Plagnol ${ }^{35}$, Lucy Raymond ${ }^{36}$, David B. Savage ${ }^{31}$ Peter J. Scambler ${ }^{3}$, Miriam Schmidts ${ }^{3}$, Robert Semple ${ }^{31}$, Eva Serra ${ }^{28}$, Jim Stalker ${ }^{28}$, Margriet van Kogelenberg ${ }^{28}$, Parthiban Vijayarangakannan ${ }^{28} \&$ Klaudia Walter ${ }^{28}$

${ }^{28}$ The Wellcome Trust Sanger Institute, Wellcome Trust Genome Campus, Hinxton CB10 1HH, Cambridge, UK. ${ }^{29}$ Department of Pathology, King Abdulaziz Medical City, Riyadh, 14611 Saudi Arabia. ${ }^{30}$ Department of Cardiovascular Medicine and Wellcome Trust Centre for Human Genetics, Roosevelt Drive, Oxford OX3 7BN, UK. ${ }^{31}$ University of Cambridge Metabolic Research Laboratories and NIHR Cambridge Biomedical Research Centre, Institute of Metabolic Science, Addenbrooke's Hospital, Cambridge CB2 OQQ, UK. ${ }^{32}$ Dubowitz Neuromuscular Centre, UCL Institute of Child Health and Great Ormond Street Hospital, London WC1N 3JH, UK. ${ }^{33}$ MRC Human Genetics Unit, MRC Institute of Genetic and Molecular Medicine, Western General Hospital, University of Edinburgh, Edinburgh EH4 2XU, UK. ${ }^{34}$ Cardiovascular Genetics, BHF Laboratories, Rayne Building, Institute Cardiovascular Sciences, University College London, London WC1E 6JJ, UK. ${ }^{35}$ University College London (UCL) Genetics Institute (UGI) Gower Street, London WC1E 6BT, UK. ${ }^{36}$ Department of Medical Genetics, Cambridge Institute for Medical Research, University of Cambridge, Cambridge CB2 2XY, UK. 